\title{
Thermodynamics of supercooled water
}

\author{
V. Holten, C. E. Bertrand, ${ }^{\text {a) }}$ M. A. Anisimov, ${ }^{\text {b) }}$ and J. V. Sengers \\ Institute for Physical Science and Technology and Department of Chemical and Biomolecular Engineering, \\ University of Maryland, College Park, Maryland 20742, USA
}

(Dated: 22 February 2013)

We review the available experimental information on the thermodynamic properties of supercooled ordinary and heavy water and demonstrate the possibility of modeling these thermodynamic properties on a theoretical basis. We show that by assuming the existence of a liquid-liquid critical point in supercooled water, the theory of critical phenomena can give an accurate account of the experimental thermodynamic-property data up to a pressure of $150 \mathrm{MPa}$. In addition, we show that a phenomenological extension of the theoretical model can account for all currently available experimental data in the supercooled region, up to $400 \mathrm{MPa}$. The stability limit of the liquid state and possible coupling between crystallization and liquid-liquid separation are discussed. It is concluded that critical-point thermodynamics describes the available thermodynamic data for supercooled water within experimental accuracy, thus establishing a benchmark for further developments in this area.

\section{INTRODUCTION}

The peculiar thermodynamic behavior of supercooled water continues to receive considerable attention, despite several decades of work in this field. Upon supercooling, water exhibits an anomalous increase of its isobaric heat capacity and its isothermal compressibility, and an anomalous decrease of its expansivity coefficient. ${ }^{1}$ One explanation of these anomalies, originally proposed by Poole et al., ${ }^{2}$ is based on the presumed existence of a liquid-liquid critical point (LLCP) in water in the deeply supercooled region. The hypothesis of the existence of a critical point in metastable water has been considered by many authors, as recently reviewed by Bertrand and Anisimov. ${ }^{3}$ In particular, several authors have made attempts to develop a thermodynamic model for the thermodynamic properties of supercooled water based on the LLCP scenario. ${ }^{3-8}$ The existence of a liquid-liquid critical point in supercooled water is still being debated, especially in view of some recent simulations. ${ }^{9,10}$ The purpose of this article is to demonstrate that a theoretical model based on the presumed existence of a second critical point in water is capable of representing, accurately and consistently, all available experimental thermodynamic property data for supercooled ordinary and heavy water. While being conceptually close to the previous works by Fuentevilla and Anisimov ${ }^{7}$ and Bertrand and Anisimov, ${ }^{3}$ this work is the first comprehensive correlation of thermodynamic properties of supercooled water, incorporating non-critical backgrounds in a thermodynamically consistent way.

This article is organized as follows. In Sec. II we review the currently available experimental information for the thermodynamic properties of supercooled water. We also provide an assessment of the IAPWS- 95 formulation ${ }^{11,12}$ for the thermodynamic properties of $\mathrm{H}_{2} \mathrm{O}$ (designed for water at temperatures above the melting temperature) when extrapolated into the supercooled region, confirming the practical need for an

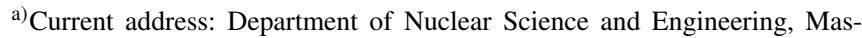
sachusetts Institute of Technology, Cambridge, Massachusetts 02139, USA

b) Author to whom correspondence should be addressed. Electronic mail: anisimov@umd.edu
}

improved equation of state for supercooled water. In Sec. III we formulate a thermodynamic model for supercooled water by adopting suitable physical scaling fields with respect to the location of a liquid-liquid critical point in supercooled water. In Sec. IV we show that this theoretical model yields an accurate representation of the thermodynamic property data of both supercooled $\mathrm{H}_{2} \mathrm{O}$ and $\mathrm{D}_{2} \mathrm{O}$ up to pressures of $150 \mathrm{MPa}$. In Sec. V we present a less-restricted phenomenological extension of the theoretical model and show that this extension allows a representation of all currently available experimental data for supercooled $\mathrm{H}_{2} \mathrm{O}$ up to the pressure of $400 \mathrm{MPa}$. The article concludes with a discussion of the results and of some unresolved theoretical issues in Sec. VI and VII.

\section{REVIEW OF EXPERIMENTAL DATA}

Experimental data on the properties of supercooled water have been reviewed by Angell in 1982,13,14 by Sato et al. in $1991,{ }^{15}$ and by Debenedetti in $2003 .{ }^{1}$ Therefore, in this review, we focus on data published after 2003, and restrict ourselves to bulk thermodynamic properties. For a complete overview of the older data the reader is referred to the earlier reviews. Besides reviewing experiments, we also assess the performance in the supercooled region of the current reference correlation for the properties of water and steam, the "IAPWS Formulation 1995 for the Thermodynamic Properties of Ordinary Water Substance for General and Scientific Use," or IAPWS-95 for short. ${ }^{11,12}$ Such an assessment has been carried out before, ${ }^{12,16}$ but not with all property data that are now available. Most of the data discussed here are provided in numerical form in the supplemental material. ${ }^{17}$

\section{A. Density}

Since the review by Debenedetti, ${ }^{1}$ new data for the density of supercooled water have been reported. ${ }^{20-22}$ Most notable is the recent work of Mishima, ${ }^{21}$ who measured the density and compressibility down to $200 \mathrm{~K}$ and up to $400 \mathrm{MPa}$; see Fig. 1(a). More accurate density measurements have been published by Sotani et al. ${ }^{19}$ Asada et al.,${ }^{20}$ and Guignon et 


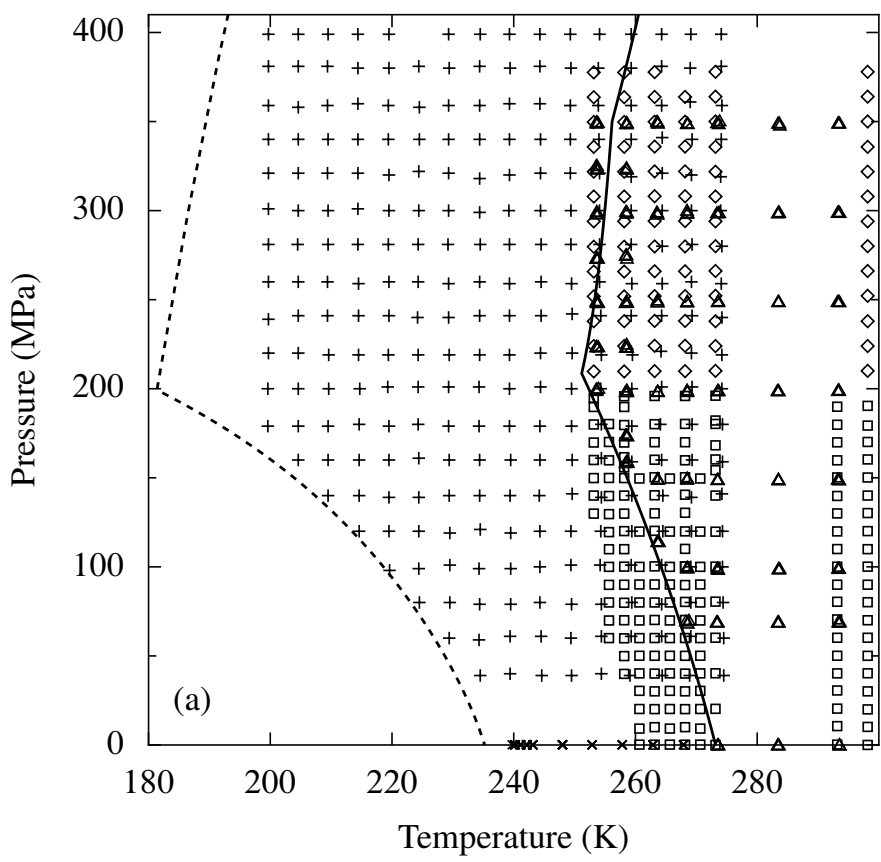

Temperature (K)

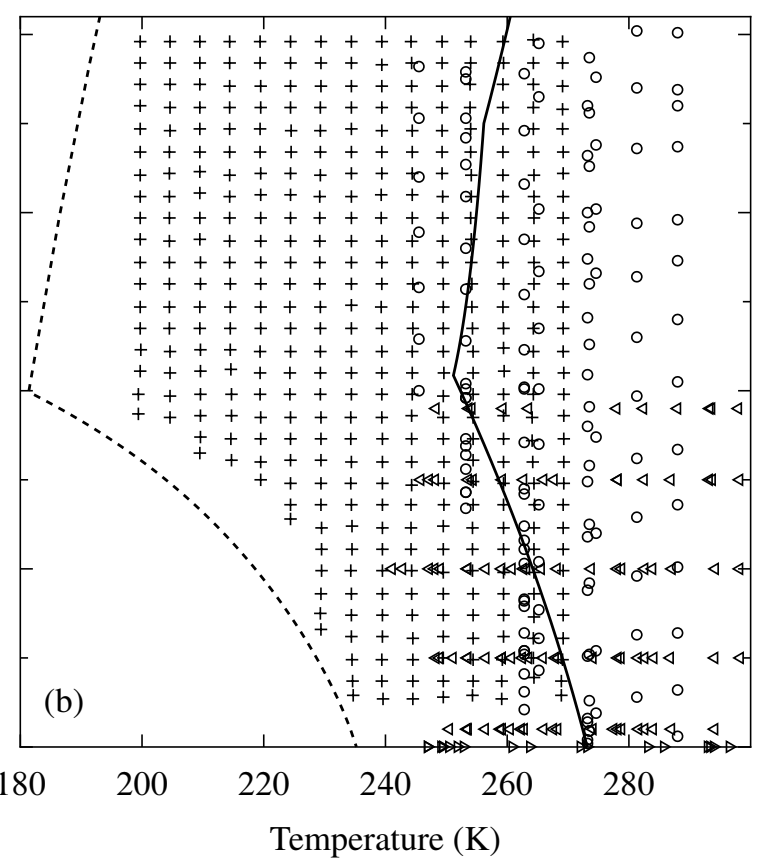

$\triangleright$ Speedy and Angell (1976) $\quad \circ$ Ter Minassian et al. (1981) Kanno and Angell (1979) + Mishima (2010)

FIG. 1. (a) Location of the experimental $\mathrm{H}_{2} \mathrm{O}$ density data. ${ }^{18-22}$ The solid curve is the ice-liquid phase boundary; ${ }^{23}$ the dashed curve is the homogeneous ice nucleation limit. ${ }^{24,25}$ The location of the dashed curve above $300 \mathrm{MPa}$ is uncertain. At $0.1 \mathrm{MPa}$ and in the stable-liquid region, data from several older sources have been omitted for clarity. (b) Location of the experimental $\mathrm{H}_{2} \mathrm{O}$ density-derivative data. Ter Minassian et $a l .{ }^{26}$ have measured the expansivity coefficient, other authors ${ }^{21,27,28}$ have measured the isothermal compressibility.

al. ${ }^{22}$ but their lowest temperature is $253 \mathrm{~K}$, so in a larger temperature range Mishima's data are the only data available. The data of Guignon et al. are in the same range as the data of Sotani et al. and Asada et al.; the maximum density difference between the data is $0.25 \%$, which is within the experimental uncertainty.

At atmospheric pressure, the density of supercooled water has been measured by several experimentalists. ${ }^{1}$ We consider the measurements of Hare and Sorensen ${ }^{18}$ of 1987 the most accurate. They showed that their measurements were not affected by the 'excess density' effect, which occurs in thin capillary tubes and caused too large densities in their 1986 experiments ${ }^{29}$ and in experiments of others.

A comparison of the densities calculated from the IAPWS95 formulation with the experimental density data of Hare and Sorensen, ${ }^{18}$ of Sotani et al. ${ }^{19}$ and of Mishima ${ }^{21}$ is shown in Fig. 2. While the IAPWS-95 formulation reproduces the experimental density data at ambient pressure, the deviations from the formulation become larger and larger with increasing pressure. Especially at higher pressures, there is a sizable discrepancy between the IAPWS-95 formulation and the experimental data; the slope (or the expansivity) even has a different sign.

\section{B. Derivatives of the density}

Both the isothermal compressibility and the expansivity coefficient of supercooled water have been measured; see Fig. 1(b). The most accurate compressibility data are from Kanno and Angell, ${ }^{28}$ whereas Mishima's ${ }^{21}$ data cover the largest temperature range. The only expansivity measurements are from Ter Minassian et al. ${ }^{26}$ Hare and Sorensen ${ }^{18,29}$ also have published expansivities (at $0.1 \mathrm{MPa}$ ), but these were obtained from the derivative of a fit to their density data.

According to Wagner and Pruß, ${ }^{12}$ the behavior of the expansivity coefficient calculated from the IAPWS-95 formulation should be reasonable in the liquid region at low temperature. However, from Fig. 3 we see that the IAPWS-95 expansivity is in error by up to $50 \%$ in the low-temperature region, even at temperatures above the melting temperature where the IAPWS-95 formulation should be valid. The isothermal compressibility calculated from the IAPWS-95 formulation agrees with the experimental data down to about $250 \mathrm{~K}$ and up to $400 \mathrm{MPa}$, as shown in Fig. 4. However, at lower temperatures, the IAPWS-95 compressibilities do not even qualitatively agree with the data.

\section{Heat capacity}

The isobaric heat capacity $C_{P}$ of supercooled water has been measured only at atmospheric pressure. ${ }^{1}$ Old measurements by Anisimov et al. ${ }^{30}$ down to $266 \mathrm{~K}$ already showed an anomalous increase of the heat capacity at moderate supercooling. In breakthrough experiments, Angell et al. ${ }^{31-33}$ ex- 


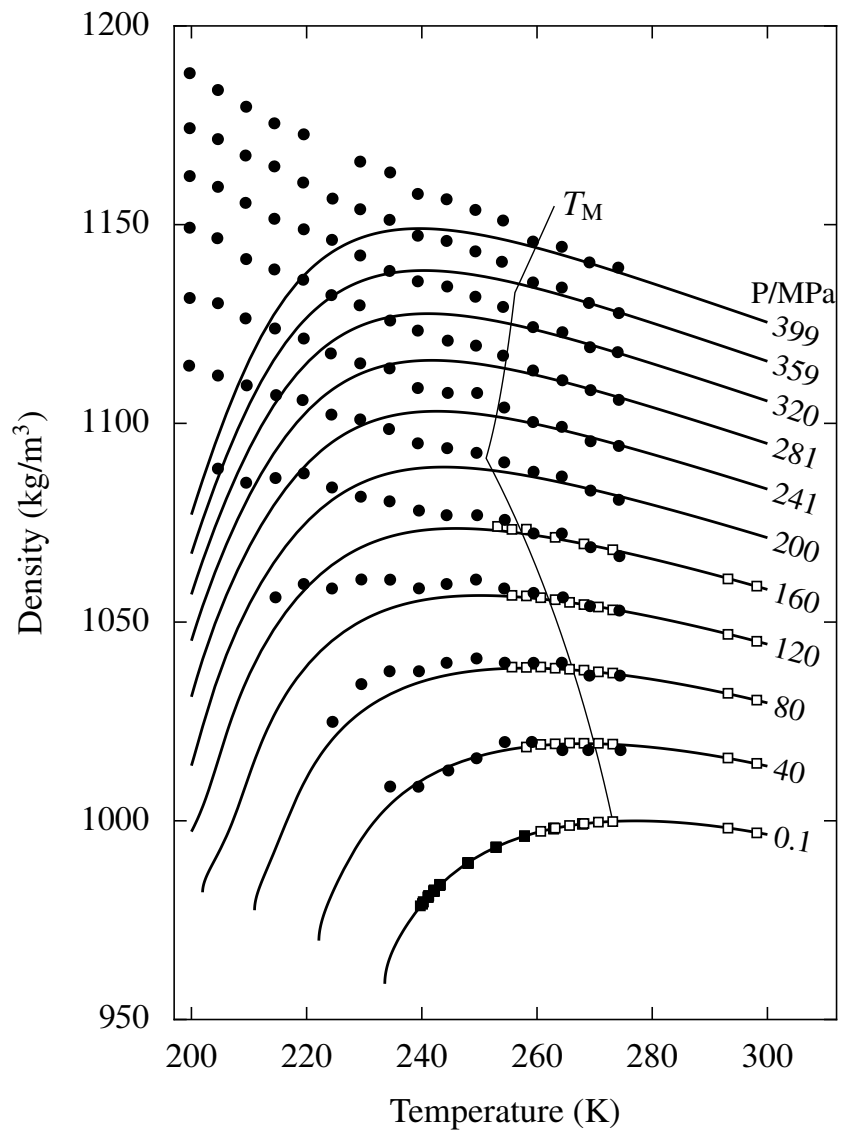

- Hare and Sorensen (1987) - Mishima (2010)

- Sotani et al. (2000) _ IAPWS-95

FIG. 2. Densities according to IAPWS-95 (curves). IAPWS-95 is valid to the right of the melting curve ${ }^{23} T_{\mathrm{M}}$; the IAPWS-95 values left of the melting curve are extrapolations. The symbols represent experimental data of Mishima, ${ }^{21}$ Sotani et al. ${ }^{19}$ and Hare and Sorensen. ${ }^{18}$ Mishima's data have been adjusted as described in Appendix C.

tended the range of measurements down to $236 \mathrm{~K}$, and demonstrated that $C_{P}$ keeps increasing with decreasing temperature (Fig. 5). More recent measurements by Archer and Carter, ${ }^{34}$ also down to $236 \mathrm{~K}$, do not perfectly agree with those of Angell et al. ${ }^{33}$ Archer and Carter suggest that the temperature calibration procedure of Angell et al. might cause a systematic error in their measurements. Furthermore, Archer and Carter suspect that measurements of Tombari et al. ${ }^{35}$ (down to $245 \mathrm{~K}$ ) could be affected by even more significant systematic calibration errors. The measurements of Bertolini et al. ${ }^{36}$ down to $247 \mathrm{~K}$ agree with those of Angell et al. (after correction, as described in Appendix C).

Because there are no high-pressure measurements of $C_{P}$ in the supercooled region, we mention here some experiments at high pressure $P$ in the stable region. Sirota et al..$^{37}$ measured $C_{P}$ up to $100 \mathrm{MPa}$ and down to $272 \mathrm{~K}$. Recently, Manyà et al. ${ }^{38}$ have measured $C_{P}$ at $4 \mathrm{MPa}$ from $298 \mathrm{~K}$ to $465 \mathrm{~K}$. It turns out that the results of Manyà et al. imply that the derivative $\left(\partial C_{P} / \partial P\right)_{T}$ at constant temperature $T$ is positive for pressures lower than $4 \mathrm{MPa}$, which contradicts the thermodynamic relation $\left(\partial C_{P} / \partial P\right)_{T}=-T\left(\partial^{2} V / \partial T^{2}\right)_{P}$, with $V$

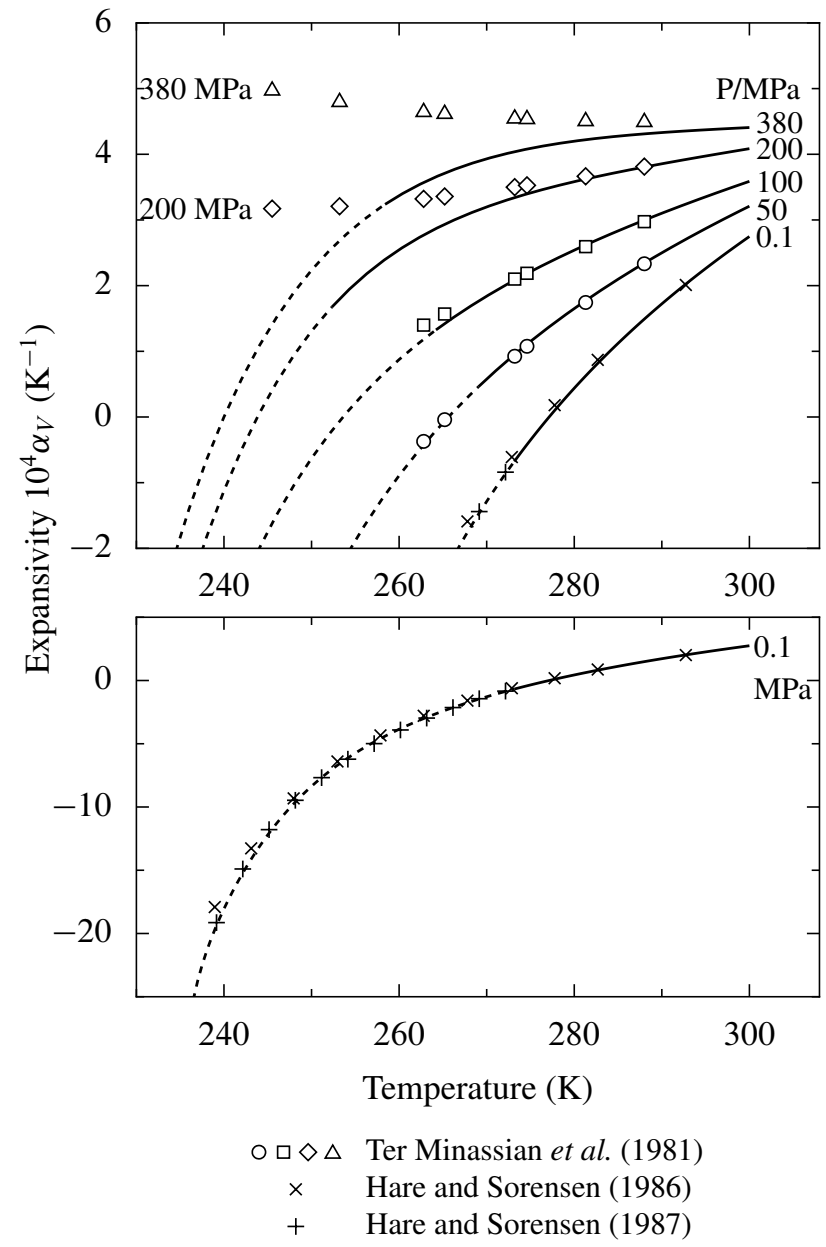

FIG. 3. Expansivity coefficient according to IAPWS-95 (solid curves: within region of validity, dashed curves: extrapolation). Symbols represent experimental data of Ter Minassian et al. ${ }^{26}$ and Hare and Sorensen. ${ }^{18,29}$

being the molar volume. Hence, the data of Manyà et al. will not be considered in this paper.

To our knowledge, the isochoric heat capacity $C_{V}$ of supercooled water has not been measured. The values presented by several investigators ${ }^{31,32,39}$ were calculated from other thermodynamic properties.

The isobaric and isochoric heat capacities predicted by IAPWS-95 at $0.1 \mathrm{MPa}$ are shown in Fig. 5 . The $C_{P}$ curve computed from IAPWS-95 follows the data of Angell et al. ${ }^{33}$ to which it was fitted. Consequently, IAPWS-95 systematically deviates from the data of Archer and Carter, ${ }^{34}$ as discussed by Wagner and Pruß. ${ }^{12}$ The isochoric heat capacity $C_{V}$ does not exhibit a strongly anomalous behavior according to the IAPWS-95 prediction.

\section{Surface tension}

As listed by Debenedetti, ${ }^{1}$ the surface tension of supercooled water with vapor and with air has been measured by Hacker, ${ }^{40}$ Floriano and Angell, ${ }^{41}$ and Trinh and Ohsaka, ${ }^{42}$ all 


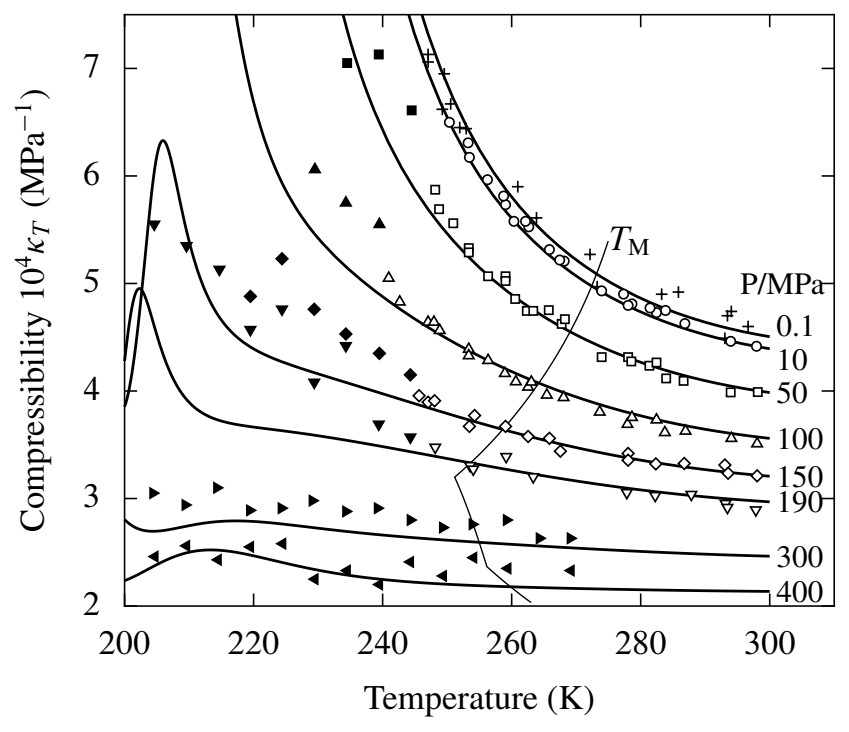

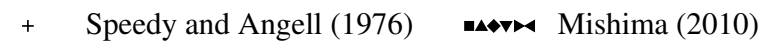
$\circ \square \Delta \diamond \nabla$ Kanno and Angell (1979)

FIG. 4. Isothermal compressibility according to IAPWS-95 (curves). IAPWS-95 is valid to the right of the melting curve $T_{\mathrm{M}}$; the IAPWS95 values left of the melting curve are extrapolations. Symbols represent experimental data of Speedy and Angell, ${ }^{27}$ Kanno and Angell, ${ }^{28}$ and Mishima. ${ }^{21}$ Solid and open symbols with the same shape correspond to the same pressure.

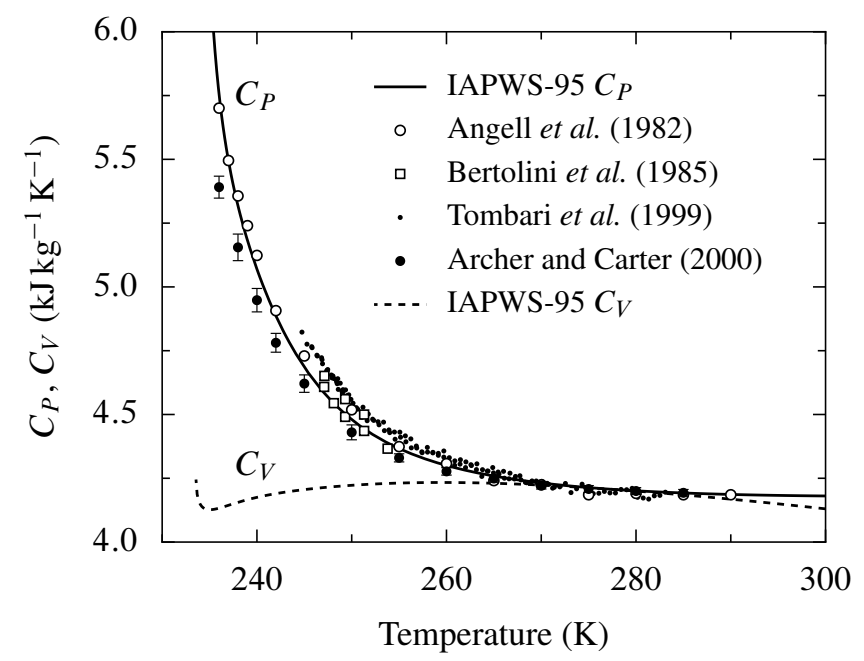

FIG. 5. Isobaric and isochoric heat capacity at $0.1 \mathrm{MPa}$ according to IAPWS-95 (curves). Symbols represent experimental data. ${ }^{33-36}$

down to about $250 \mathrm{~K}$. Hacker's measurements show an inflection point at about $268 \mathrm{~K}$, below which the surface tension has a stronger temperature dependence (Fig. 6). The data of Floriano and Angell are less accurate, but the authors also noted an inflection. The measurements of Trinh and Ohsaka show a systematic deviation from the other data, but the trend agrees with Hacker's data. Furthermore, a molecular dynamics simulation by Lü and $\mathrm{Wei}^{43}$ shows an inflection point as well.

IAPWS has recommended an equation to represent the sur-

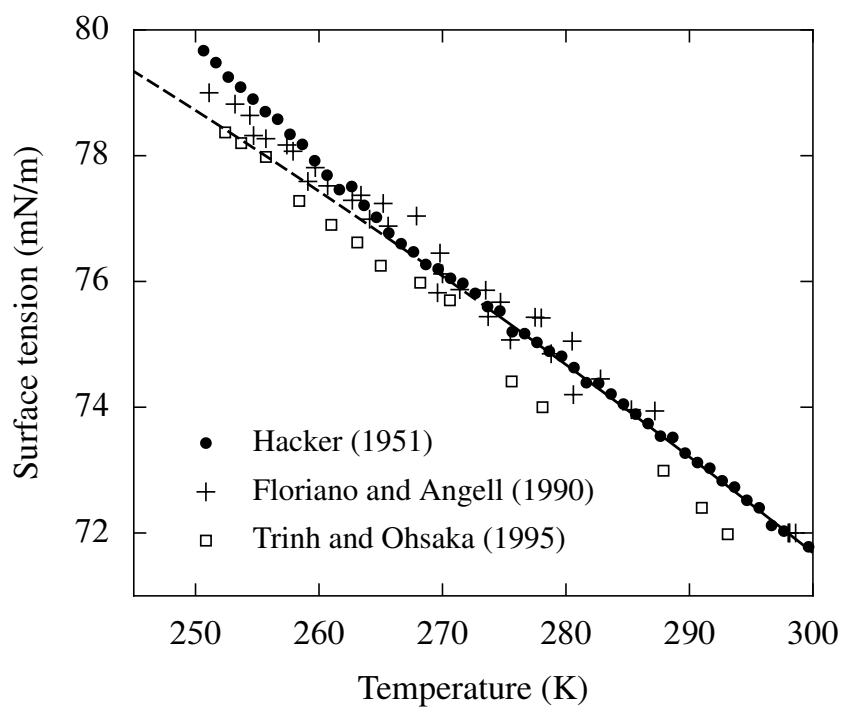

FIG. 6. Surface tension according to the IAPWS equation ${ }^{44}$ (solid curve: within region of validity, dashed curve: extrapolation). Symbols represent experimental data from Hacker, ${ }^{40}$ Floriano and Angell, ${ }^{41}$ and Trinh and Ohsaka. ${ }^{42}$

face tension of liquid water in equilibrium with water vapor. ${ }^{44}$ For a comparison with experimental data, it is necessary to consider the conditions under which the surface tension was measured. Hacker, ${ }^{40}$ Floriano and Angell, ${ }^{41}$ and Trinh and Ohsaka $^{42}$ measured the surface tension at atmospheric pressure in air. The influence of air at atmospheric pressure on the surface tension of water is usually neglected, ${ }^{45}$ but for supercooled water the effect may be significant since the solubility of nitrogen and oxygen in water increases with decreasing temperature. From high-pressure data ${ }^{46}$ it is known that the surface tension of water with nitrogen or oxygen is lower than the pure-water surface tension. In Fig. 6 we show a comparison of the experimental surface-tension data with the values calculated from the IAPWS equation. As noted above, the experimental values of the surface tension suggest an inflection point at about $268 \mathrm{~K}$. The extrapolation of the IAPWS equation does not show an inflection, and the difference with Hacker's accurate data ${ }^{40}$ increases with decreasing temperature. The data of Floriano and Angell ${ }^{41}$ show more scatter, but below the freezing point most of their data lie above the IAPWS extrapolation. The measurements of Trinh and Ohsaka ${ }^{42}$ lie below all other data; the deviation is about the same as their experimental uncertainty, which is $1 \%$.

\section{E. Speed of sound}

The speed of sound in supercooled water has been measured in a broad frequency range, from $54 \mathrm{kHz}$ to about $20 \mathrm{GHz}$. In stable water at atmospheric pressure, no significant dependence on the frequency is found in this range, but the speed of sound in supercooled water shows a dispersion that increases with cooling. In this article we consider the thermodynamic (zero-frequency) limit of the speed of sound, which 


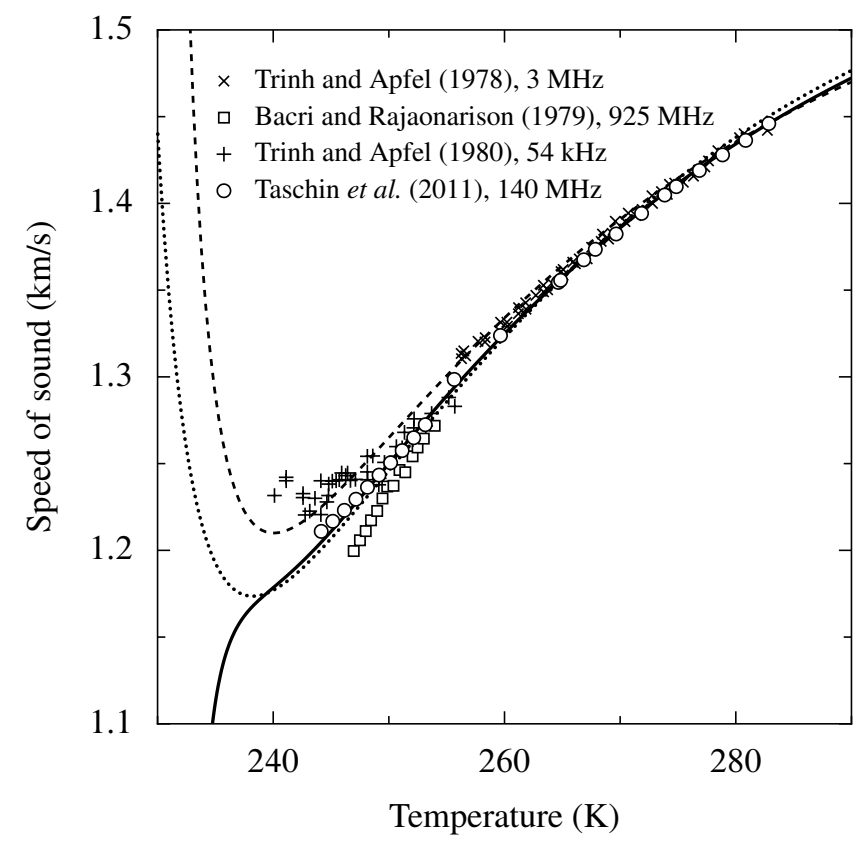

FIG. 7. Speed of sound at $0.1 \mathrm{MPa}$. Solid curve: prediction of the IAPWS-95 formulation. Dashed curve: model of Sec. IV; dotted curve: model of Sec. V. Symbols represent experimental data. ${ }^{47,53,54,57,58}$

is associated with the adiabatic compressibility. ${ }^{13}$ Taschin $e t$ $a l^{47}$ estimate that dispersion effects become noticeable at frequencies of $1 \mathrm{GHz}$ and higher. We will therefore not consider the measurements in the $1-10 \mathrm{GHz}$ range obtained with Brillouin light scattering, which show a speed-of-sound minimum between $250 \mathrm{~K}$ and $280 \mathrm{~K}$; the temperature of the minimum increases with increasing frequency. ${ }^{48,49}$ The lowerfrequency (ultrasonic) measurements are shown in Fig. 7. Below $255 \mathrm{~K}$, there appears to be dispersion; however, the speed of sound decreases with increasing frequency, which suggests an anomalous (negative) dispersion. While a negative dispersion of the speed of sound very close to the vapor-liquid critical point is, in principle, possible due to the divergence of the thermal conductivity, ${ }^{50}$ there is not yet any experimental indication for an anomaly of the thermal conductivity of supercooled water. ${ }^{51}$ Recent simulations of Kumar and Stanley ${ }^{52}$ show a minimum for this property. Debenedetti ${ }^{1}$ argues that negative dispersion can be ruled out because the Brillouin experiments all show positive dispersion. According to Taschin et al. ${ }^{47}$ the apparent negative dispersion is a result of systematic errors in the data of Trinh and $\mathrm{Apfe}^{53}$ and Bacri and Rajaonarison. ${ }^{54}$ The IAPWS-95 formulation agrees with the recent data of Taschin et al. ${ }^{47}$ to within their uncertainty.

There are no measurements of the speed of sound of supercooled water at high pressure. In the stable region, Petitet et $a l .{ }^{55}$ performed measurements at $10 \mathrm{MHz}$ up to $460 \mathrm{MPa}$, including the region close to the melting curve, down to $253 \mathrm{~K}$. Petitet et al. have also published data down to $253 \mathrm{~K}$ at atmospheric pressure, but these data were taken from Conde $e t$ $a l .{ }^{56}$ Since Conde et al. measured at $5 \mathrm{GHz}$, these data do not represent the zero-frequency limit of the speed of sound.

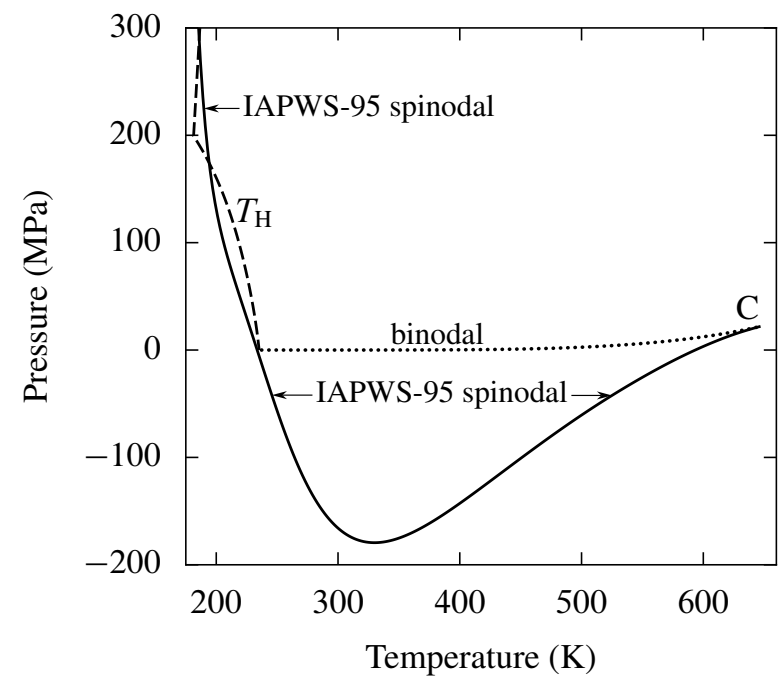

FIG. 8. Location of the liquid spinodal according to the IAPWS95 formulation. The curved marked with $T_{\mathrm{H}}$ is the homogeneous ice nucleation limit. ${ }^{24,25}$ Also shown is the location of the binodal, the vapor pressure curve, and $\mathrm{C}$ denotes the vapor-liquid critical point.

\section{F. Spinodal}

In the supercooled region, IAPWS-95 predicts a re-entrant liquid spinodal, as shown in Fig. 8. The spinodal pressure becomes positive at $233.6 \mathrm{~K}$, which is a few degrees below the homogeneous nucleation limit. At about $195 \mathrm{~K}$ and $175 \mathrm{MPa}$, the spinodal curve crosses the homogeneous nucleation limit and enters the region where supercooled water can be experimentally observed. Up to about $290 \mathrm{MPa}$, the spinodal curve stays in the experimentally accessible range. Since a spinodal has not been observed there, the spinodal of IAPWS-95 contradicts experimental evidence. While a re-entrant spinodal is thermodynamically possible, Debenedetti has argued that the re-entrant spinodal scenario suggested by Speedy ${ }^{59}$ is implausible for supercooled water. ${ }^{1,60}$ Debenedetti's argument may be summarized as follows. In the pressure-temperature plane (Fig. 8), a re-entrant spinodal must intersect the metastable continuation of the binodal, the vapor-pressure curve. At the intersection, liquid water is simultaneously in equilibrium with water vapor and unstable with respect to infinitesimal density fluctuations. Since these two conditions are mutually exclusive, such an intersection cannot exist. On the other hand, at the vapor-liquid critical point the binodal and spinodal do coincide, which is possible because the vapor and liquid phases are indistinguishable there.

\section{G. Liquid-liquid coexistence curve}

Both the existence of a second critical point and its location are still being debated in the literature. If the second critical point exists, there should be a liquid-liquid transition (LLT) curve - separating a hypothetical high-density liquid and lowdensity liquid - which ends at the critical point. At pressures below the critical pressure, water's response functions exhibit 


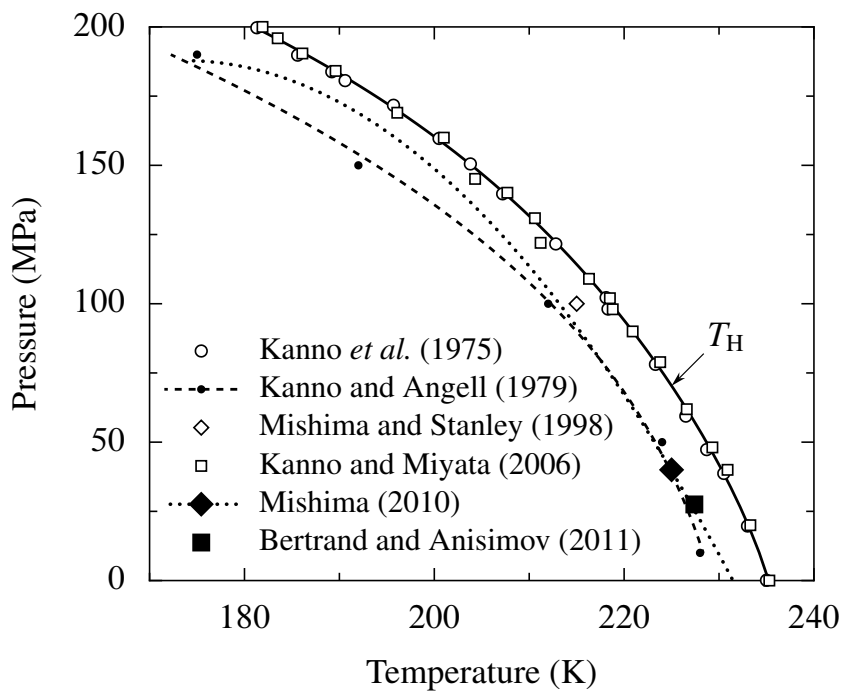

FIG. 9. Homogeneous ice nucleation temperatures (open circles ${ }^{24}$ and squares ${ }^{25}$ and fitted solid curve), Mishima's ${ }^{21}$ conjectured liquid-liquid coexistence curve (dotted) and liquid-liquid critical point (large solid diamond), and Kanno and Angell's curve (dashed) connecting the fitted singular temperatures (solid circles). ${ }^{28}$ Open diamond: bend in the melting curve of ice IV ${ }^{61}$ large solid square: the liquid-liquid critical point suggested by Bertrand and Anisimov. ${ }^{3}$

an extremum near the 'Widom line,' which is the extension of the LLT curve into the one-phase region and the locus of maximum fluctuations of the order parameter.

While the location of the critical point obtained by different simulations varies greatly, different attempts to locate the LLT and the Widom line from experimental data have yielded approximately the same result. Kanno and Angell ${ }^{28}$ fitted empirical power laws to their compressibility measurements and obtained singular temperatures located from $5 \mathrm{~K}$ to $12 \mathrm{~K}$ below the homogeneous nucleation temperature $T_{\mathrm{H}}$ (Fig. 9), suggesting a LLT that mimics the $T_{\mathrm{H}}$ curve but shifted to lower temperature. Mishima measured metastable melting curves of $\mathrm{H}_{2} \mathrm{O}$ ice IV $\mathrm{V}^{61}$ and $\mathrm{D}_{2} \mathrm{O}$ ices IV and $\mathrm{V},{ }^{62}$ and found that they suddenly bent at temperatures of $4 \mathrm{~K}$ to $7 \mathrm{~K}$ below $T_{\mathrm{H}}$. According to Mishima, this is indirect evidence for the location of the LLT, but a one-to-one correspondence between a break in the melting curve and the LLT has been questioned by Imre and Rzoska. ${ }^{63}$

Mishima $^{21}$ approximated the LLT by a quadratic function of $T$ with approximately the same shape as the $T_{\mathrm{H}}$ curve, but allowing a shift to lower temperature. His final result, shown in Fig. 9, is close to Kanno and Angell's curve for pressures up to $100 \mathrm{MPa}$, albeit with a different curvature.

\section{H. Heavy water}

Experimental data on the properties of supercooled heavy water $\left(\mathrm{D}_{2} \mathrm{O}\right)$ have been reviewed by Angell ${ }^{13,14}$ and Debenedetti. ${ }^{1}$ The location of data for the density and its derivatives is shown in Fig. 10. At atmospheric pressure, the density has been measured by several investigators. ${ }^{29,65,67,69}$

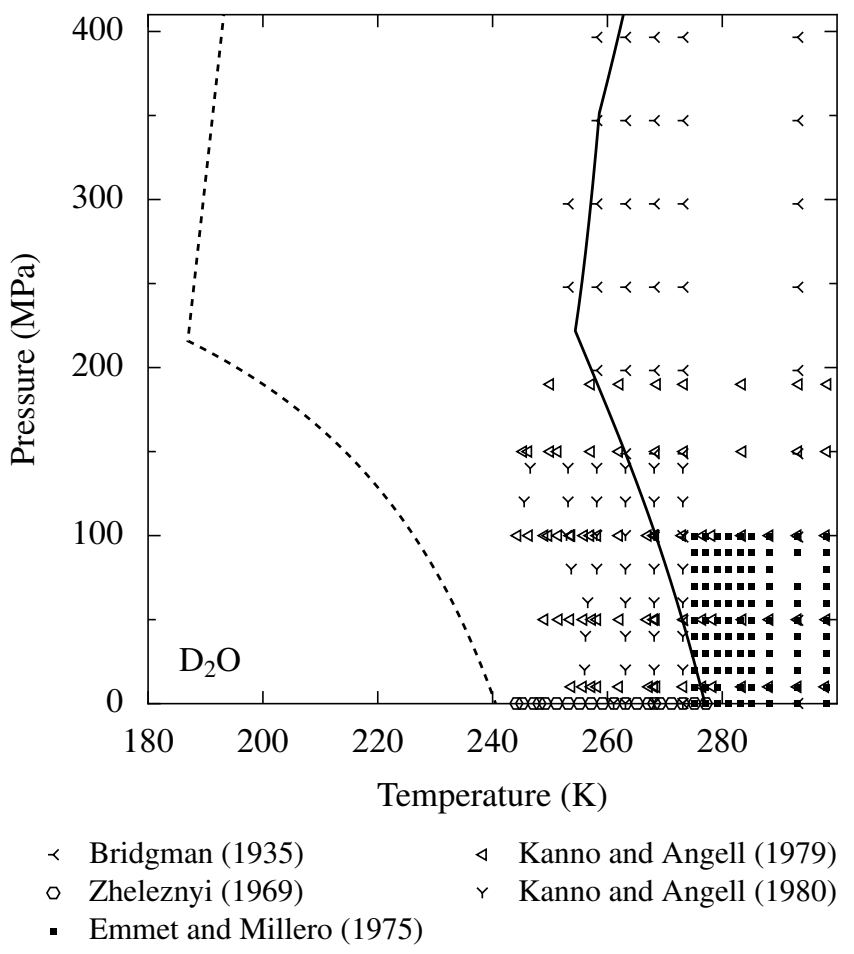

FIG. 10. (a) Location of the experimental data for the density of $\mathrm{D}_{2} \mathrm{O}$ and its derivatives. ${ }^{28,64-67}$ The solid curve is the ice-liquid phase boundary; ${ }^{64}$ the dashed curve is the homogeneous ice nucleation limit. ${ }^{68}$ The location of the dashed curve above $300 \mathrm{MPa}$ is uncertain. At $0.1 \mathrm{MPa}$ and in the stable-liquid region, data from several sources have been omitted for clarity.

There are differences of up to $0.14 \%$ between the data sets, and it is not clear which is the best set. The isothermal compressibility and thermal expansion coefficient have been measured in an extensive temperature and pressure range by Kanno and Angell. ${ }^{28,67}$ The isobaric heat capacity has been measured at atmospheric pressure by Angell and coworkers. ${ }^{31-33}$ The speed of sound of supercooled $\mathrm{D}_{2} \mathrm{O}$ was reported by Conde et al. ${ }^{56}$ at $5 \mathrm{GHz}$; at this frequency the speed of sound likely deviates from the zero-frequency limit, especially at lower temperatures.

\section{THERMODYNAMIC MODEL FOR SUPERCOOLED WATER}

In this section we further develop a scaling model for bulk thermodynamic properties supercooled water, which was earlier suggested by Fuentevilla and Anisimov ${ }^{7}$ and, more recently, modified and elaborated by Bertrand and Anisimov. ${ }^{3}$

\section{A. Scaling fields and thermodynamic properties}

Fluids belong to the universality class of Ising-like systems whose critical behavior is characterized by two independent scaling fields, a "strong" scaling field $h_{1}$ (ordering field) and a "weak" scaling field $h_{2}$, and by a dependent scaling field 
$h_{3}$ which asymptotically close to the critical point becomes a generalized homogeneous function of $h_{1}$ and $h_{2}:^{70-72}$

$$
h_{3}\left(h_{1}, h_{2}\right) \approx\left|h_{2}\right|^{2-\alpha} f^{ \pm}\left(\frac{h_{1}}{\left|h_{2}\right|^{2-\alpha-\beta}}\right) .
$$

In this expression $\alpha \simeq 0.110$ and $\beta \simeq 0.326$ are universal critical exponents ${ }^{73,74}$ and $f^{ \pm}$, with the superscripts \pm referring to $h_{2}>0$ and $h_{2}<0$, is a universal scaling function except for two system-dependent amplitudes. Associated with these scaling fields are two conjugate scaling densities, a strongly fluctuating scaling density $\phi_{1}$ (order parameter) and a weakly fluctuating scaling density $\phi_{2}$, such that

$$
\mathrm{d} h_{3}=\phi_{1} \mathrm{~d} h_{1}+\phi_{2} \mathrm{~d} h_{2}
$$

with

$$
\phi_{1}=\left(\frac{\partial h_{3}}{\partial h_{1}}\right)_{h_{2}}, \quad \phi_{2}=\left(\frac{\partial h_{3}}{\partial h_{2}}\right)_{h_{1}} .
$$

In addition one can define three susceptibilities, a "strong" susceptibility $\chi_{1}$, a "weak" susceptibility $\chi_{2}$, and a "cross" susceptibility $\chi_{12}$ :

$$
\begin{gathered}
\chi_{1}=\left(\frac{\partial \phi_{1}}{\partial h_{1}}\right)_{h_{2}}, \quad \chi_{2}=\left(\frac{\partial \phi_{2}}{\partial h_{2}}\right)_{h_{1}}, \\
\chi_{12}=\left(\frac{\partial \phi_{1}}{\partial h_{2}}\right)_{h_{1}}=\left(\frac{\partial \phi_{2}}{\partial h_{1}}\right)_{h_{2}} .
\end{gathered}
$$

In fluids and fluid mixtures one encounters a large variety of different types of critical phenomena. ${ }^{75}$ The asymptotic thermodynamic behavior near all kinds of critical points can be described in terms of Eq. (1). The differences arise from the actual relationships between the scaling fields and the physical fields, ${ }^{76}$ subject to the condition that at the critical point

$$
h_{1}=h_{2}=h_{3}=0 .
$$

In one-component fluids the relevant physical fields are the chemical potential $\mu$ (Gibbs energy per mole), the temperature $T$, and the pressure $P$. To satisfy condition (6) one defines $\Delta \mu=\mu-\mu_{\mathrm{c}}, \Delta T=T-T_{\mathrm{c}}$, and $\Delta P=P-P_{\mathrm{c}}$. In this article we adopt the usual convention that a subscript $\mathrm{c}$ refers to the value of the property at the critical point. There are two special models for critical behavior that deserve some attention. The first is the lattice gas in which the ordering field $h_{1}$ is asymptotically proportional to $\Delta \mu$ and the weak scaling field proportional to $\Delta T \cdot{ }^{77-79}$ Hence, in the lattice gas $\phi_{1}$ is proportional to $\Delta \rho=\rho-\rho_{\mathrm{c}}$ and $\phi_{2}$ proportional to $\Delta s=s-s_{\mathrm{c}}$, where $\rho$ is the molar density and $s$ the entropy density. The lattice gas provides a model for the vapor-liquid critical point where the molar density yields the major contribution to the order parameter. In practice, the asymptotic critical behavior of a fluid near the vapor-liquid critical point, including that of $\mathrm{H}_{2} \mathrm{O},{ }^{80}$ can be described by a slight modification of the latticegas model to account for some lack of vapor-liquid symmetry in real fluids. Another special model, introduced by Bertrand and Anisimov, ${ }^{3}$ is a "lattice liquid" in which the ordering field is asymptotically proportional to $\Delta T$ and in which the weak scaling field is proportional to $\Delta \mu .^{3}$ Near the liquid-liquid critical point in weakly compressible supercooled water the entropy yields the major contribution to the order parameter and not the mass density, as first pointed out by Fuentevilla and Anisimov. ${ }^{7}$ Thus the thermodynamic properties near this liquid-liquid critical point can be described by a slight modification of the lattice-liquid model to account for some lack of symmetry in the order parameter. ${ }^{3}$

To implement a scaled thermodynamic representation it is convenient to make all thermodynamic properties dimensionless in terms of the critical parameters $T_{\mathrm{c}}$ and $\rho_{\mathrm{c}}$ or $V_{\mathrm{c}}=\rho_{\mathrm{c}}^{-1}$ :

$$
\hat{T}=\frac{T}{T_{\mathrm{c}}}, \quad \hat{\mu}=\frac{\mu}{R T_{\mathrm{c}}}, \quad \hat{P}=\frac{P V_{\mathrm{c}}}{R T_{\mathrm{c}}},
$$

where $R$ is the ideal-gas constant. For the dimensionless physical densities we define

$$
\hat{V}=\frac{V}{V_{\mathrm{c}}}, \quad \hat{S}=\frac{S}{R}, \quad \hat{C}_{P}=\frac{C_{P}}{R},
$$

where $V$ is the molar volume, $S$ the molar entropy, and $C_{P}$ the isobaric molar heat capacity. The thermodynamic model of Bertrand and Anisimov was formulated in terms of $\hat{P}(\hat{\mu}, \hat{T})$ for which

$$
\mathrm{d} \hat{P}=\hat{V}^{-1} \mathrm{~d} \hat{\mu}+\hat{V}^{-1} \hat{S} \mathrm{~d} \hat{T} .
$$

We have found it more convenient, and practically equivalent, to formulate this thermodynamic model in terms of $\hat{\mu}(\hat{P}, \hat{T})$ for which

$$
\mathrm{d} \hat{\mu}=\hat{V} \mathrm{~d} \hat{P}-\hat{S} \mathrm{~d} \hat{T} .
$$

Thus in this formulation, similar to that suggested earlier by Fuentevilla and Anisimov, ${ }^{7}$ we identify the order parameter with the entropy itself instead of the entropy density. In our model the scaling fields are related to the physical fields as

$$
\begin{aligned}
& h_{1}=\Delta \hat{T}+a^{\prime} \Delta \hat{P}, \\
& h_{2}=-\Delta \hat{P}+b^{\prime} \Delta \hat{T}, \\
& h_{3}=\Delta \hat{P}-\Delta \hat{\mu}+\Delta \hat{\mu}^{\mathrm{r}},
\end{aligned}
$$

with

$$
\Delta \hat{T}=\frac{T-T_{\mathrm{c}}}{T_{\mathrm{c}}}, \quad \Delta \hat{P}=\frac{\left(P-P_{\mathrm{c}}\right) V_{\mathrm{c}}}{R T_{\mathrm{c}}}, \quad \Delta \hat{\mu}=\frac{\mu-\mu_{\mathrm{c}}}{R T_{\mathrm{c}}} .
$$

In Eq. (11) $a^{\prime}$ represents the slope $-\mathrm{d} \hat{T} / \mathrm{d} \hat{P}$ of the phasecoexistence or Widom line at the critical point. In Eq. (12) $b^{\prime}$ is a so-called mixing coefficient which accounts for the fact that the critical phase transition in supercooled water is not completely symmetric in terms of the entropy order parameter. Introduction of mixing of this type is also known in the literature as the revised-scaling approximation. ${ }^{81}$ Equation (1) only represents the asymptotic behavior of the so-called singular critical contributions to the thermodynamic properties. To obtain a complete representation of the thermodynamic properties we need to add a regular (i.e., analytic) background contribution. As has been common practice in developing scaled equations 
of state in fluids near the vapor-liquid critical point, ${ }^{80,81}$ the regular background contribution is represented by a truncated Taylor-series expansion around the critical point:

$$
\Delta \hat{\mu}^{\mathrm{r}}=\sum_{m, n} c_{m n}(\Delta \hat{T})^{m}(\Delta \hat{P})^{n}, \quad \text { with } \quad c_{00}=c_{10}=c_{01}=0 .
$$

The first two terms in the temperature expansion of $\Delta \hat{\mu}^{\mathrm{r}}$ depend on the choice of zero entropy and energy and do not appear in the expressions of any of the physically observable thermodynamic properties. Hence, these coefficients may be set to zero. Furthermore, the coefficient $c_{01}=\hat{V}_{\mathrm{c}}-1=0$. Strictly speaking, critical fluctuations also yield an analytic contribution to $h_{3} .{ }^{82,83}$ In this article we incorporate this contribution into the linear background contribution as has also been done often in the past.

The current treatment of the background contribution differs from that in earlier publications. ${ }^{3,7}$ Previously, a temperature-dependent background was added to the critical part of each property. Because the background of each property was treated separately, the resulting property values were not mutually consistent. In this work, the background is added to the chemical potential, and the backgrounds in the derived properties follow, ensuring thermodynamic consistency.

From the fundamental thermodynamic differential relation (10) it follows that

$$
\begin{aligned}
& \hat{V}=\left(\frac{\partial \hat{\mu}}{\partial \hat{P}}\right)_{T}=1-a^{\prime} \phi_{1}+\phi_{2}+\Delta \hat{\mu}_{\hat{P}}^{\mathrm{r}}, \\
& \hat{S}=-\left(\frac{\partial \hat{\mu}}{\partial \hat{T}}\right)_{P}=\phi_{1}+b^{\prime} \phi_{2}-\Delta \hat{\mu}_{\hat{T}}^{\mathrm{r}} .
\end{aligned}
$$

In this article we adopt the convention that a subscript $\hat{P}$ indicates a derivative with respect to $\hat{P}$ at constant $\hat{T}$ and a subscript $\hat{T}$ a derivative with respect to $\hat{T}$ at constant $\hat{P}$. Finally, the dimensionless isothermal compressibility $\hat{\kappa}_{T}$, expansivity coefficient $\hat{\alpha}_{V}$, and isobaric heat capacity $\hat{C}_{P}$ can be expressed in terms of the scaling susceptibilities $\chi_{1}, \chi_{2}$, and $\chi_{12}$ :

$$
\begin{aligned}
\hat{\kappa}_{T}= & -\frac{1}{\hat{V}}\left(\frac{\partial \hat{V}}{\partial \hat{P}}\right)_{T}=\frac{1}{\hat{V}}\left[\left(a^{\prime}\right)^{2} \chi_{1}+\chi_{2}-2 a^{\prime} \chi_{12}-\Delta \hat{\mu}_{\hat{P} \hat{P}}^{\mathrm{r}}\right] \\
\hat{\alpha}_{V} & =\frac{1}{\hat{V}}\left(\frac{\partial \hat{V}}{\partial \hat{T}}\right)_{P} \\
& =\frac{1}{\hat{V}}\left[-a^{\prime} \chi_{1}+b^{\prime} \chi_{2}+\left(1-a^{\prime} b^{\prime}\right) \chi_{12}+\Delta \hat{\mu}_{\hat{T} \hat{P}}^{\mathrm{r}}\right] \\
\hat{C}_{P}= & \hat{T}\left(\frac{\partial \hat{S}}{\partial \hat{T}}\right)_{P}=\hat{T}\left[\chi_{1}+\left(b^{\prime}\right)^{2} \chi_{2}+2 b^{\prime} \chi_{12}-\Delta \hat{\mu}_{\hat{T} \hat{T}}^{\mathrm{r}}\right]
\end{aligned}
$$

\section{B. Parametric equation of state}

It is not possible to write the scaled expression (1) for $h_{3}$ as an explicit function of $h_{1}$ and $h_{2}$. Such attempts always cause singular behavior of the thermodynamic potential in the onephase region either at $h_{1}=0$ or at $h_{2}=0$. This problem is solved by replacing the two independent scaling fields, $h_{1}$ and $h_{2}$, with two parametric variables: a variable $r$ which measures a "distance" from the critical point and an angular variable $\theta$ which measures the location on a contour of constant $r$. A transformation most frequently adopted has the form:

$$
h_{1}=a r^{2-\alpha-\beta} \theta\left(1-\theta^{2}\right), \quad h_{2}=r\left(1-b^{2} \theta^{2}\right) .
$$

From Eqs. (1) and (3) it then follows that the order parameter $\phi_{1}$ must have the form: ${ }^{84}$

$$
\phi_{1}=k r^{\beta} M(\theta),
$$

where $M(\theta)$ is a universal analytic function of $\theta$. In principle, this function can be calculated from the renormalizationgroup theory of critical phenomena. ${ }^{85}$ In practice one adopts an analytic approximant for $M(\theta)$, the simplest one being $M(\theta)=\theta:{ }^{86}$

$$
\phi_{1}=k r^{\beta} \theta .
$$

Equations (21) and (23) define what is known as the "linear model" parametric equation of state. In these equations $a$ and $k$ are two system-dependent amplitudes related to the two system-dependent amplitudes in Eq. (1), while $b^{2}$ is a universal constant which is often approximated by ${ }^{87}$

$$
b^{2}=\frac{2-\alpha-4 \beta}{(2-\alpha-2 \beta)(1-2 \beta)} \simeq 1.361 .
$$

Equations (21) and (23) with the specific choice (24) for $b^{2}$ is known as the "restricted" linear model. ${ }^{79}$ The resulting parametric equations for the various thermodynamic properties can be found in many publications. ${ }^{79-81,83,88,89}$ In this paper we are using the "restricted" linear model to describe the supercooled-water anomalies. The parametric equations needed for the analysis in this article are listed in Appendix A.

\section{COMPARISON WITH EXPERIMENTAL DATA}

The scaling theory, formulated in the preceding section, represents the thermodynamic behavior asymptotically close to the critical point. Specifically, the liquid-liquid (LLT) curve that follows from Eq. (11) is a straight line, while the actual LLT curve should exhibit curvature (as shown in Fig. 9). In this section we investigate the thermodynamic properties of supercooled liquid water in a range of pressures and temperatures, where the asymptotic theory appears to be adequate. Issues related to nonasymptotic features of the scaling theory will be addressed in Sec. V. Hence, we restrict the asymptotic theoretical model to pressures not exceeding $150 \mathrm{MPa}$, where the LLT can be reasonably approximated by a single straight line. The slope of the LLT was constrained to values that are close to the slopes of the curves of Kanno and Angell $^{28}$ and Mishima ${ }^{21}$ in the range of $0 \mathrm{MPa}$ to $150 \mathrm{MPa}$. Specifically, the value of $a^{\prime}$ in Eq. (11) was restricted to the range of 0.065 to 0.090 . Because the position of the LLT is not precisely known, the critical point was allowed to deviate up to $3 \mathrm{~K}$ from Mishima's curve. It was found that the results of the model were rather insensitive to the critical pressure $P_{\mathrm{c}}$, so $P_{\mathrm{c}}$ was constrained to the value of $27.5 \mathrm{MPa}$ obtained 
TABLE I. Parameter values for $\mathrm{H}_{2} \mathrm{O}$ in the model of Sec. IV

\begin{tabular}{|c|c|c|c|}
\hline Parameter & Value $^{\mathrm{a}}$ & Parameter & Value \\
\hline$T T_{\mathrm{c}} / \mathrm{K}$ & 224.23 & $c_{11}$ & $1.5363 \times 10^{-1}$ \\
\hline$P_{\mathrm{c}} / \mathrm{MPa}$ & 27.5 & $c_{12}$ & $-6.4879 \times 10^{-3}$ \\
\hline$\rho_{\mathrm{c}} /\left(\mathrm{kg} \mathrm{m}^{-3}\right)$ & 948.77 & $c_{13}$ & $7.7090 \times 10^{-3}$ \\
\hline$a$ & 0.22924 & $c_{20}$ & $-3.8888 \times 10^{0}$ \\
\hline$k$ & 0.37704 & $c_{21}$ & $1.7347 \times 10^{-1}$ \\
\hline$a^{\prime}$ & 0.090 & $c_{22}$ & $-6.4157 \times 10^{-2}$ \\
\hline$c_{02}$ & $7.1779 \times 10^{-2}$ & $c_{23}$ & $-6.9850 \times 10^{-3}$ \\
\hline$c_{03}$ & $-4.0936 \times 10^{-4}$ & $c_{30}$ & $6.9813 \times 10^{-1}$ \\
\hline$c_{04}$ & $-1.0996 \times 10^{-3}$ & $c_{31}$ & $-1.1459 \times 10^{-1}$ \\
\hline$c_{05}$ & $2.9497 \times 10^{-4}$ & $c_{32}$ & $7.5006 \times 10^{-2}$ \\
\hline
\end{tabular}

${ }^{\text {a }}$ Final digits of parameter values are given to allow reproducing the values of properties with the model but do not have physical significance.

TABLE II. Parameter values for $\mathrm{D}_{2} \mathrm{O}$ in the model of Sec. IV

\begin{tabular}{lclc}
\hline \hline Parameter & \multicolumn{1}{c}{ Value } & Parameter & Value \\
\hline$T_{\mathrm{c}} / \mathrm{K}$ & 232.65 & $c_{11}$ & $1.2828 \times 10^{-1}$ \\
$P_{\mathrm{c}} / \mathrm{MPa}$ & 32.29 & $c_{12}$ & $-1.6267 \times 10^{-3}$ \\
$\rho_{\mathrm{c}} /\left(\mathrm{kg} \mathrm{m}^{-3}\right)$ & 1055.74 & $c_{13}$ & $9.5552 \times 10^{-3}$ \\
$a$ & 0.22924 & $c_{20}$ & $-4.4118 \times 10^{0}$ \\
$k$ & 0.37704 & $c_{21}$ & $3.0002 \times 10^{-1}$ \\
$a^{\prime}$ & 0.078757 & $c_{22}$ & $-9.7204 \times 10^{-2}$ \\
$c_{02}$ & $6.9072 \times 10^{-2}$ & $c_{23}$ & $-1.4402 \times 10^{-2}$ \\
$c_{03}$ & $1.7651 \times 10^{-4}$ & $c_{30}$ & $8.4968 \times 10^{-1}$ \\
$c_{04}$ & $-1.4458 \times 10^{-3}$ & $c_{31}$ & $-2.7188 \times 10^{-1}$ \\
$c_{05}$ & $4.3335 \times 10^{-4}$ & $c_{32}$ & $1.4418 \times 10^{-1}$ \\
\hline \hline
\end{tabular}

by Bertrand and Anisimov. ${ }^{3}$ It was also found that a nonzero mixing coefficient $b^{\prime}$ did not significantly improve the fit, so $b^{\prime}$ was set to zero. This means physically that the liquid-critical behavior in supercooled water exhibits little asymmetry in the order parameter and is indeed very close to lattice-liquid behavior.

Changes in the third decimal place of the values of the critical exponents $\alpha$ and $\beta$ result in small density changes that are of the order of $0.1 \%$. However, some of the density measurements for water are more accurate than $0.1 \%$; for example, the accuracy of the data of Hare and Sorensen ${ }^{18}$ and Sotani et $a l .{ }^{19}$ is $0.01 \%$. Therefore, the values of the critical exponents must be given with at least four decimal places. We have adopted the values of Pelissetto and Vicari ${ }^{73}$ and have set $\alpha=0.1100$ and $\beta=0.3265$. The value for the molar mass of $\mathrm{H}_{2} \mathrm{O}(18.015268 \mathrm{~g} / \mathrm{mol})$ was taken from Wagner and Pruß ${ }^{12}$ and the ideal-gas constant $R\left(8.3144621 \mathrm{~J} \mathrm{~mol}^{-1} \mathrm{~K}^{-1}\right)$ was taken from Mohr et al. ${ }^{90}$ The molar mass of $\mathrm{D}_{2} \mathrm{O}(20.027508$ $\mathrm{g} / \mathrm{mol}$ ) was taken from an IAPWS guideline. ${ }^{91}$

The number of terms in the background $\Delta \hat{\mu}^{\mathrm{r}}$ [Eq. (15)] was increased step by step until the experimental data could be accurately represented. The final background contains fourteen free parameters. The reason for the many background terms of higher order in temperature and pressure is that the response functions are second derivatives of the thermodynamic potential. To obtain, for example, a background term in the compressibility of second order in pressure, it is necessary to have a fourth-order pressure-dependent term in the potential. The terms in the backgrounds for each property are at most third order in temperature or in pressure. We want to emphasize that the observed anomalies are indeed due to the critical part of the equation of state since the nonlinear contributions to the regular part are needed only when the maximum pressure considered is higher than about $100 \mathrm{MPa}$. Besides the background parameters, there are five additional parameters to be determined: the critical temperature $T_{\mathrm{c}}$ and volume $V_{\mathrm{c}}$, the linearmodel amplitudes $a$ and $k$, and the slope of the LLT line $a^{\prime}$. As noted, the values of $T_{\mathrm{c}}$ and $a^{\prime}$ were constrained to a limited range.

The model was fitted to heat-capacity data of Archer and $\mathrm{Carter}^{34}$ and IAPWS-95, expansivity data of Hare and Sorensen, ${ }^{18}$ IAPWS-95 and Ter Minassian, ${ }^{26}$ compressibility data of Speedy and Angell, ${ }^{27}$ Kanno and Angell ${ }^{28}$ and Mishima, ${ }^{21}$ density data of Hare and Sorensen, ${ }^{18}$ Sotani et al. ${ }^{19}$ IAPWS-95 and Mishima, ${ }^{21}$ and speed-of-sound data of Taschin et al. ${ }^{47}$ We have made small adjustments to the data of Mishima as described in Appendix C. For all quantities except the heat capacity, values from IAPWS-95 were only used at $0.1 \mathrm{MPa}$ and above $273 \mathrm{~K}$. For the heat capacity, IAPWS-95 values in the range of $273 \mathrm{~K}$ to $305 \mathrm{~K}$ up to $100 \mathrm{MPa}$ were used. (We considered heat-capacity values calculated from IAPWS-95 more reliable than high-pressure heat-capacity data of Sirota et al..$^{37}$ ) To reduce the time needed for optimization, not all data points were used in the fitting process; about 60 points were selected for each of the quantities heat capacity, expansivity, compressibility, and density. The selected data are given in the supplemental material. ${ }^{17}$

The model was optimized by minimizing the sum of squared residuals, where the residual is the difference between model and experiment, divided by the experimental uncertainty. ${ }^{92}$ For some data the uncertainty was not given and had to be estimated. The resulting optimized parameters are listed in Tables I and II. The value of $a^{\prime}$ for $\mathrm{H}_{2} \mathrm{O}$ is exactly 0.09 because $a^{\prime}$ was restricted to the range of 0.065 to 0.090 , and the optimum is located at the edge of this range. The fitted models are valid up to $300 \mathrm{~K}$ and from 0 to $150 \mathrm{MPa}$.

As can be seen in Fig. 11, the model represents the experimental density data well. The density jumps at low temperature because the isobars cross the LLT curve there. In Fig. 12, the temperature of maximum density is plotted as a function of pressure, both for the model and for the IAPWS-95 formulation. At pressures higher than about $60 \mathrm{MPa}$, the values of IAPWS-95 deviate from the experimental data, while the current model agrees with the data (except at negative pressures, where the model was not fitted to any experimental data). The curve of the temperature of maximum density does not intersect the homogeneous nucleation curve at its break point at about $200 \mathrm{MPa}$ and $180 \mathrm{~K}$. Such an intersection was expected by Dougherty. ${ }^{96}$

The agreement of the compressibility data with values of the model is shown Fig. 13. An interesting feature is the intersection of the isobars of $0.1 \mathrm{MPa}$ and $10 \mathrm{MPa}$ at about $250 \mathrm{~K}$. The experimental data do not confirm or rule out such an intersection because of the scatter and the lack of data below $245 \mathrm{~K}$. However, the intersection implies that the pressure derivative of the compressibility, $\left(\partial \kappa_{T} / \partial P\right)_{T}$, is positive at 


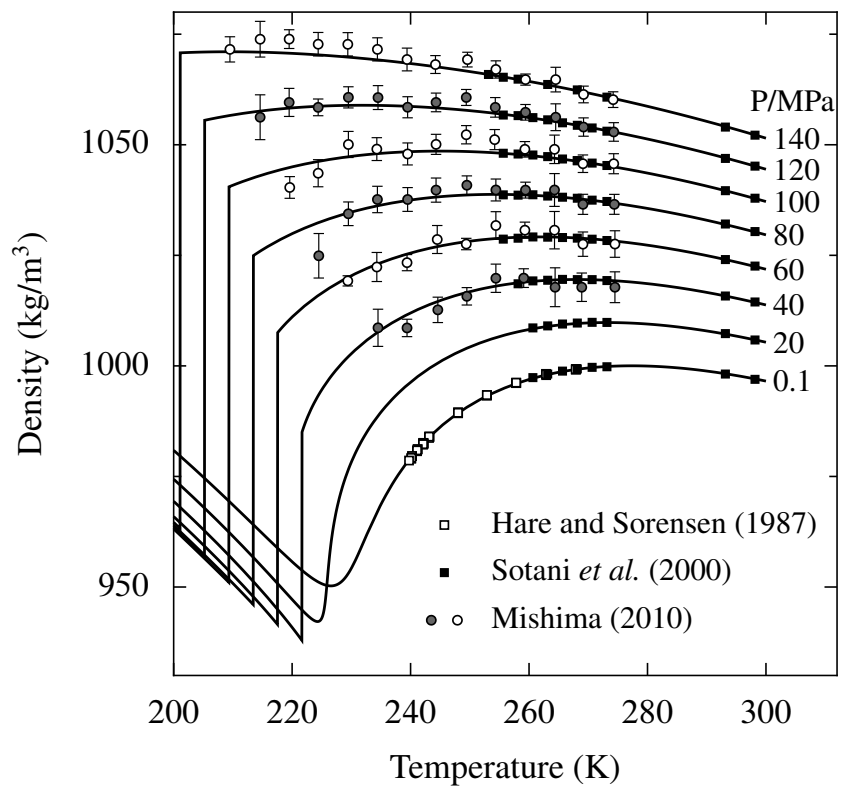

FIG. 11. Densities of $\mathrm{H}_{2} \mathrm{O}$ according to the model (curves). The symbols represent experimental data of Mishima, ${ }^{21}$ Sotani et al. ${ }^{19}$ and Hare and Sorensen. ${ }^{18}$ The symbols for Mishima's densities on different isobars are alternatingly open and filled to guide the eye.

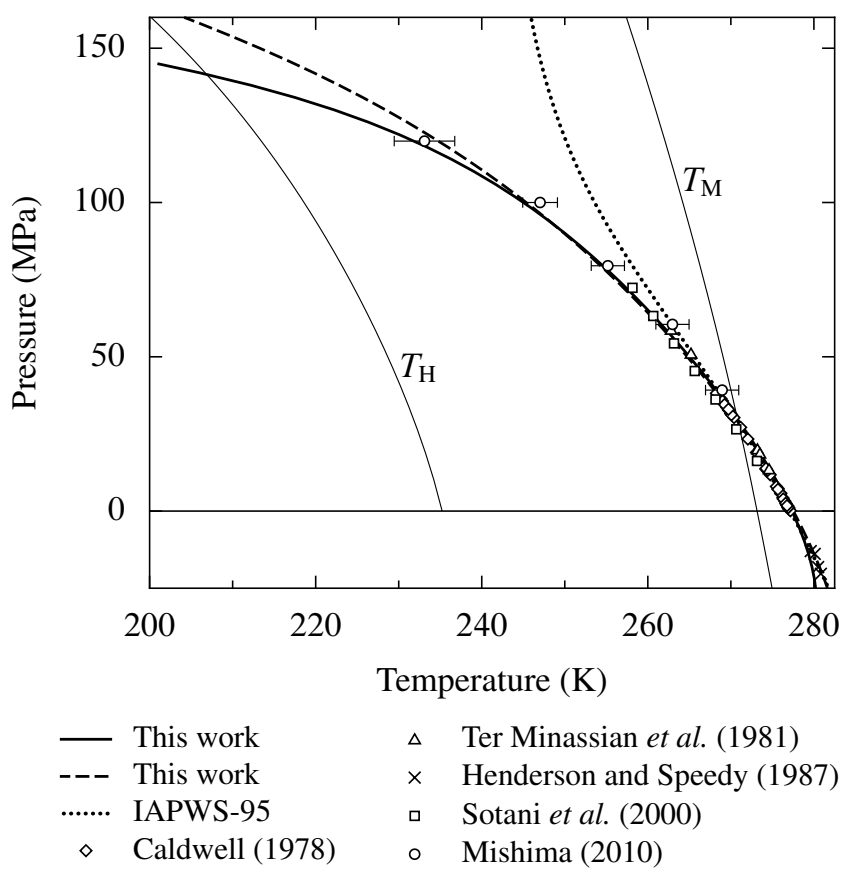

FIG. 12. Temperature of maximum density of $\mathrm{H}_{2} \mathrm{O}$ as a function of pressure according to the model of Sec. IV (thick solid curve), the model of Sec. V (dashed) and IAPWS-95 (dotted). $T_{\mathrm{M}}$ marks the melting curve ${ }^{23}$ and its extension to negative pressures; $;{ }^{93} T_{\mathrm{H}}$ denotes the homogeneous nucleation limit. ${ }^{24}$ Symbols represent experimental data. ${ }^{19,21,26,94,95}$ The temperatures of maximum density for Mishima's data ${ }^{21}$ were determined by locating the maxima of fits to his density data. low temperature and ordinary pressures.

Figure 14 shows experimental data for the expansivity coefficient and the values predicted by the model for five pressures. The model follows the experimental data, contrary to the IAPWS-95 formulation. At $240 \mathrm{~K}$, where the difference between Hare and Sorensen's data of 1986 and 1987 is largest, the expansivity predicted by the model lies between them.

Heat-capacity data are compared with the model's predictions in Figs. 15 and 16. In Fig. 15, it is seen that the model follows the data of Archer and Carter, ${ }^{34}$ whereas IAPWS-95 follows the data of Angell et al., ${ }^{33}$ to which it was fitted. However, the curvature of the $0.1 \mathrm{MPa}$ isobar of the model is slightly higher than that suggested by the data of Archer and Carter. Murphy and Koop ${ }^{97}$ proposed a heat-capacity curve with a broader peak than that of our model, but with about the same maximum value. The predicted isochoric heat capacity $C_{V}$ diverges, as will be discussed in Sec. VIC. Figure 16 shows the heat capacity as a function of pressure. There is a systematic difference between the data of Sirota et al. ${ }^{37}$ in the stable region and the values of IAPWS-95, and the data of Sirota et al. were not selected for the fit of the current model. At $250 \mathrm{~K}$ and pressures above about $50 \mathrm{MPa}$, the model predicts a smaller pressure dependence of the heat capacity than IAPWS-95. The pressure dependence of the heat capacity is thermodynamically related to the expansivity coefficient, and we have seen that the expansivity coefficient of IAPWS-95 does not agree with experimental data at low temperature and high pressure (see Fig. 14). Therefore, differences between the heat-capacity values of the current model and IAPWS-95 are to be expected.

The speed of sound predicted by the model is shown in Fig. 7. The prediction agrees fairly well with the experimental data. At $240 \mathrm{~K}$, the model shows a minimum in the speed of sound whereas IAPWS-95 predicts a monotonically decreasing speed of sound with decreasing temperature. The divergence of the speed of sound of the model at low temperature is related to a stability limit as will be discussed in Sec. VIC.

For heavy water, the model was fitted to heat-capacity data of Angell et al. ${ }^{33}$ and Zábranský et al., ${ }^{98}$ expansivity data of Kell ${ }^{99}$ and Kanno and Angell, ${ }^{67}$ compressibility data of Kanno and Angell, ${ }^{28}$ density data of Kell, ${ }^{99}$ Zheleznyi, ${ }^{65}$ and Rasmussen and MacKenzie, ${ }^{69}$ and speed-of-sound data of Chen and Millero ${ }^{100}$ and Marczak. ${ }^{101}$ It has been found that in the equation of state for $\mathrm{H}_{2} \mathrm{O}$ and $\mathrm{D}_{2} \mathrm{O}$ near the vapor-liquid critical point deviations from corresponding states only appear in the analytic background contributions, while the amplitudes of the scaling fields are identical in accordance with corresponding states. ${ }^{102,103}$ Thus also for the second critical point we assigned to the linear-model amplitudes $a$ and $k$ the same values for $\mathrm{D}_{2} \mathrm{O}$ as for $\mathrm{H}_{2} \mathrm{O}$.

Figures 17-22 show the predictions of the model for heavy water compared with experimental data on density, compressibility, expansion coefficient, heat capacity, and speed of sound. The results are similar to those for ordinary water. The quality of the description is almost as good as for ordinary water, except for the speed of sound; the experimental data for speed of sound show noticeable (a few percent) deviation from the model. The expansivity data of Zheleznyi, ${ }^{65}$ shown in Fig. 20, deviate from the prediction of the model 


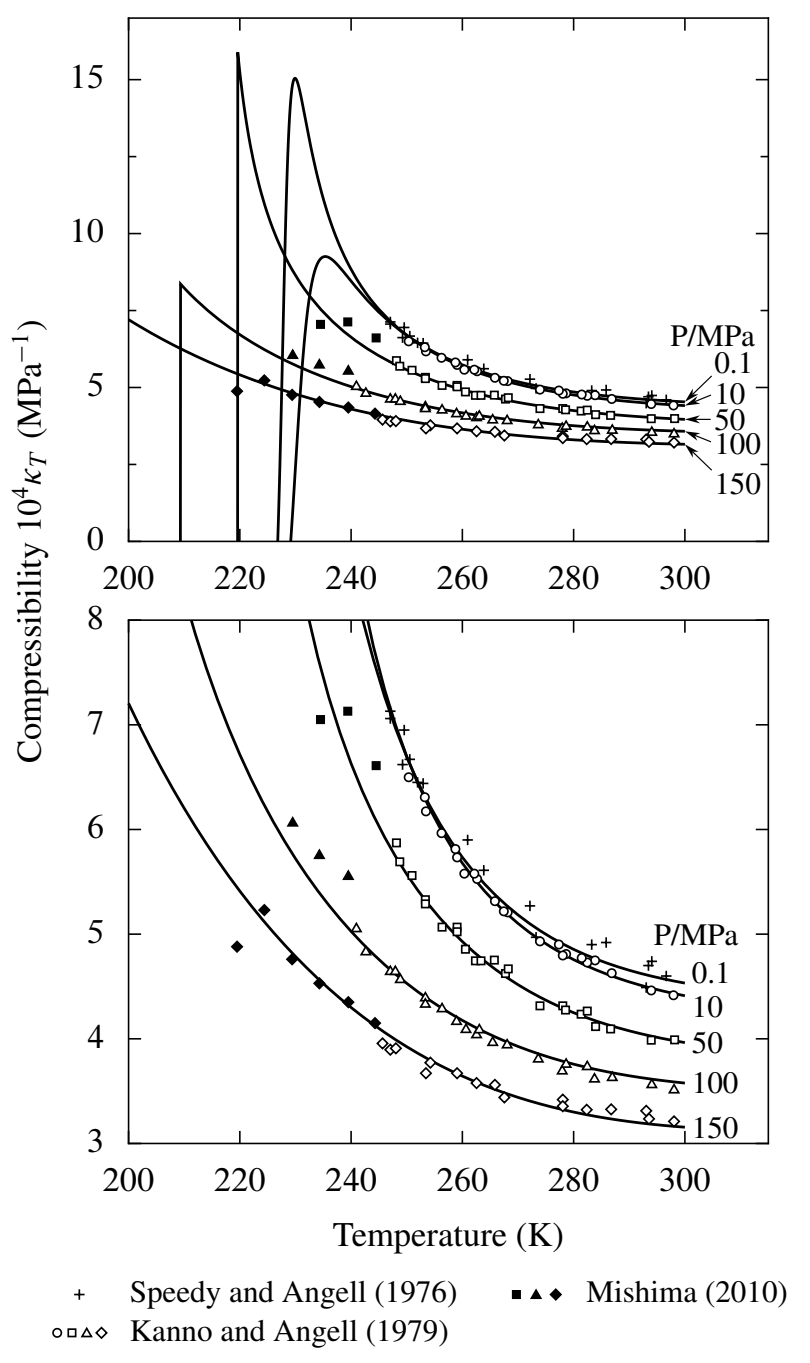

FIG. 13. Isothermal compressibility of $\mathrm{H}_{2} \mathrm{O}$ according to the model (curves). For clarity, the curves are not shown for temperatures below the LLT line in the bottom graph. Symbols represent experimental data of Speedy and Angell, ${ }^{27}$ Kanno and Angell, ${ }^{28}$ and Mishima. ${ }^{21}$ Solid and open symbols with the same shape correspond to the same pressure.

below about $255 \mathrm{~K}$. For ordinary water, Hare and Sorensen ${ }^{18}$ concluded that the data of Zheleznyi "show smaller densities for $T<-25^{\circ} \mathrm{C}$ than our bulk data and thus overestimate the anomaly in the expansivity." For heavy water, the density and expansivity values of Zheleznyi are also likely too low, since the densities measured by others ${ }^{29,67,69}$ are all higher.

While the critical parts of the thermodynamic properties of $\mathrm{H}_{2} \mathrm{O}$ and $\mathrm{D}_{2} \mathrm{O}$ follow the law of corresponding states (the critical amplitudes $a$ and $k$ are the same) the corresponding regular parts do not follow this law. The critical compressibility factors $Z_{\mathrm{c}}=P_{\mathrm{c}} /\left(\rho_{\mathrm{c}} R T_{\mathrm{c}}\right)$ for $\mathrm{H}_{2} \mathrm{O}\left(Z_{\mathrm{c}}=0.28\right)$ and $\mathrm{D}_{2} \mathrm{O}$ $\left(Z_{\mathrm{c}}=0.32\right)$ LLCP differ by about $13 \%$ while for the vaporliquid critical point these factors differ by only $1 \% .{ }^{103}$ The difference of $13 \%$ should be taken too seriously since the fit of the model is insensitive to the value of $P_{\mathrm{c}}$ at the LLCP.

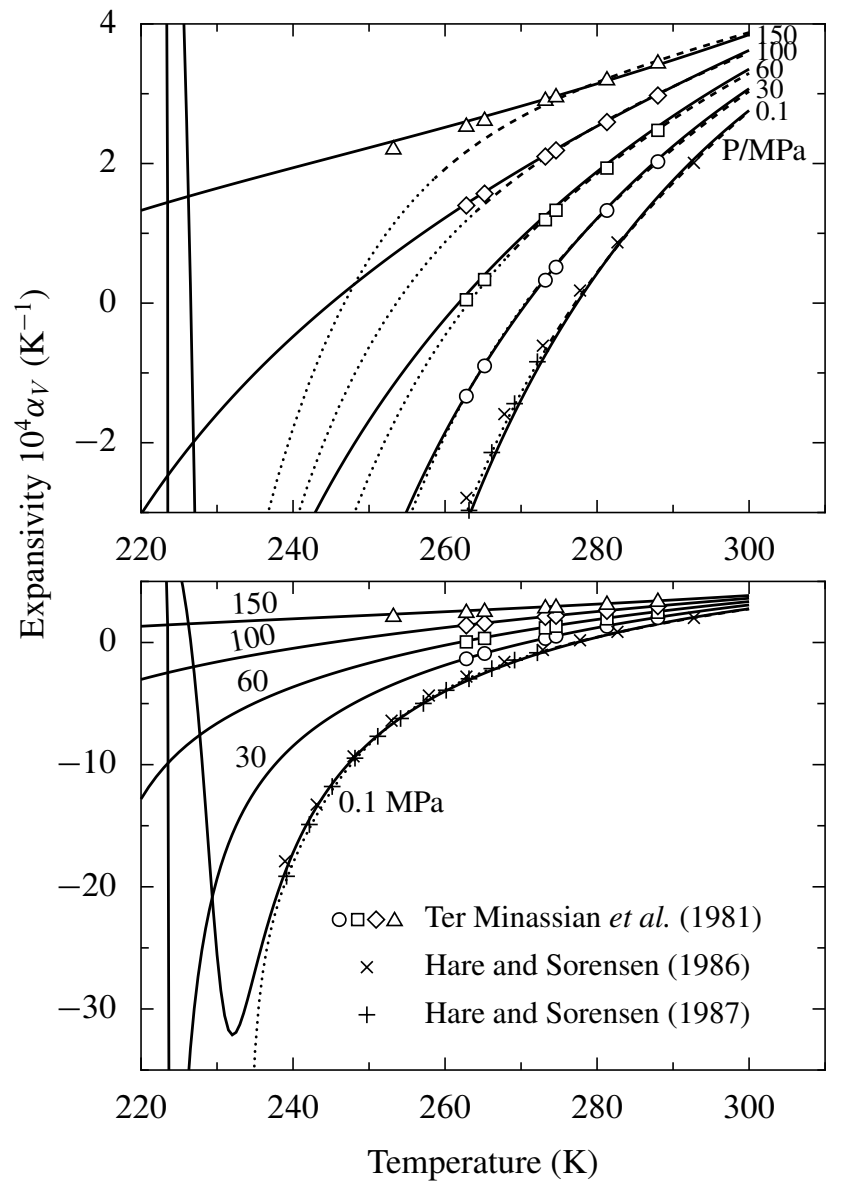

FIG. 14. Expansivity coefficient of $\mathrm{H}_{2} \mathrm{O}$ according to the model (solid curves) and IAPWS-95 (dashed: within region of validity, dotted: extrapolations). Symbols represent experimental data of Ter Minassian et al. ${ }^{26}$ and Hare and Sorensen. ${ }^{18,29}$

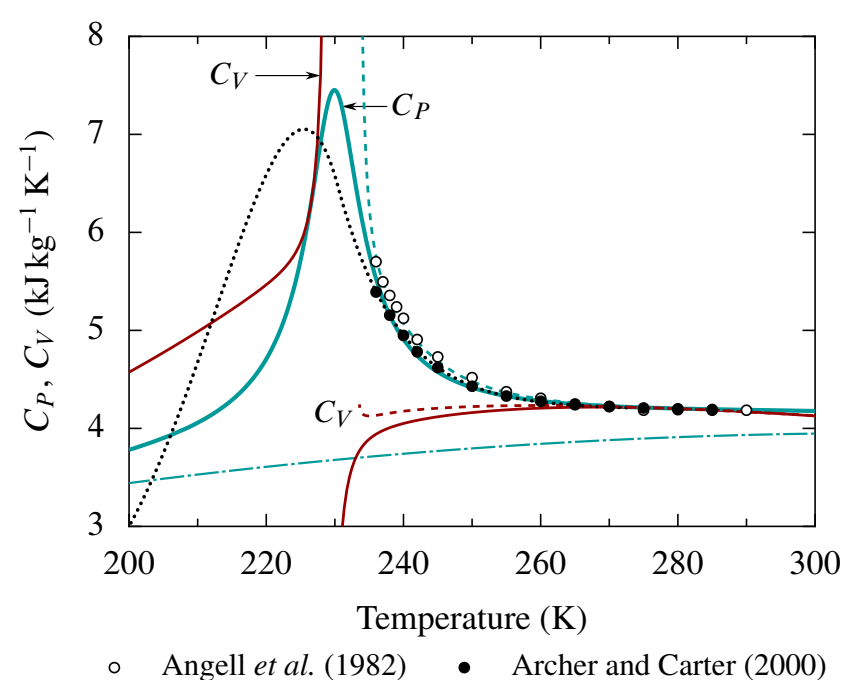

FIG. 15. Isobaric and isochoric heat capacity of $\mathrm{H}_{2} \mathrm{O}$ versus temperature at $0.1 \mathrm{MPa}$ according to the model (solid curves), IAPWS-95 (dashed), and the prediction of Murphy and Koop (dotted). Symbols represent experimental data of Angell et al. ${ }^{33}$ and Archer and Carter. ${ }^{34}$ The dash-dotted line is the estimated regular part of $C_{P}$. The predicted thermodynamic behavior of $C_{V}$ is discussed in Sec. VIC. 


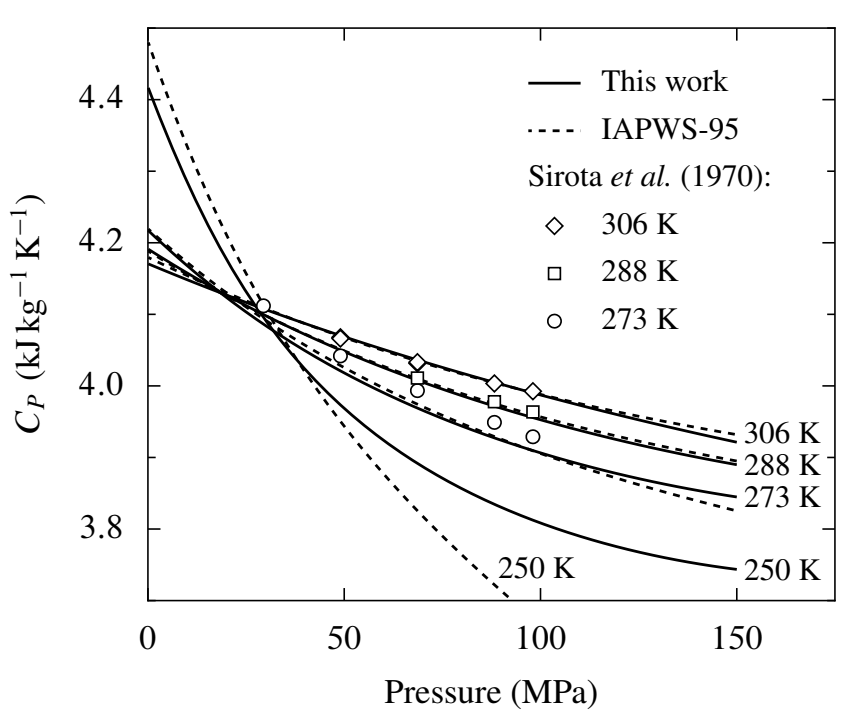

FIG. 16. Isobaric heat capacity of $\mathrm{H}_{2} \mathrm{O}$ versus pressure according to the model (solid curves) and IAPWS-95 (dashed). Symbols represent experimental data of Sirota et al. ${ }^{37}$

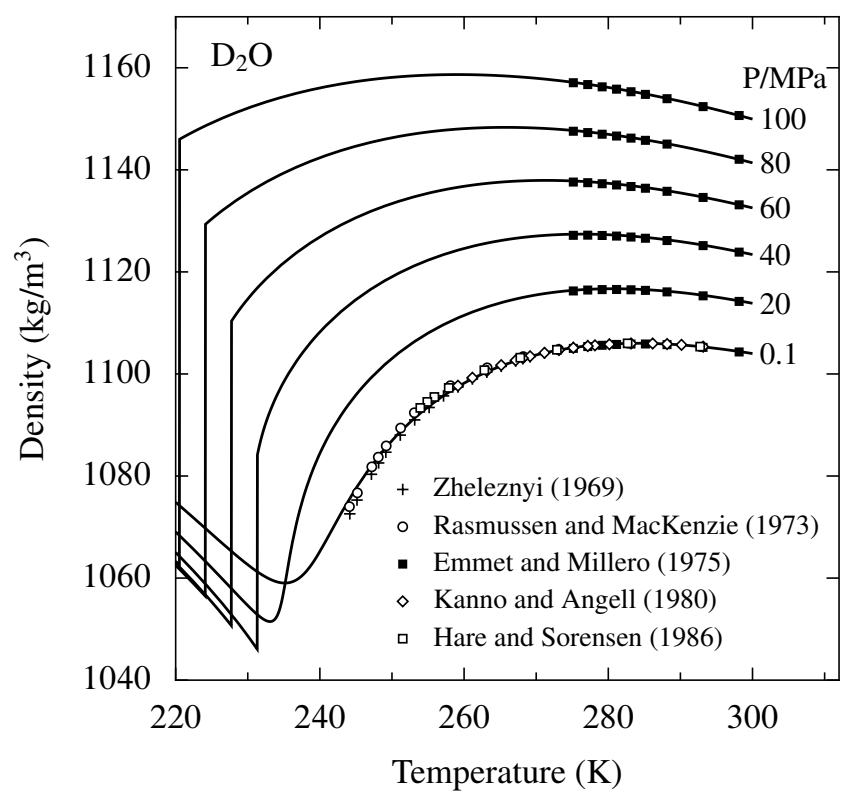

FIG. 17. Density of $\mathrm{D}_{2} \mathrm{O}$ according to the model (curves). The symbols represent experimental data. ${ }^{29,65-67,69}$

\section{SEMI-EMPIRICAL EXTENSION OF SCALING MODEL}

As mentioned in the preceding section, the asymptotic theoretical model implies a linear LLT line. An attempt to include an additional term accounting for curvature of the LLT curve has been made by Fuentevilla and Anisimov ${ }^{7}$ by introducing a pressure-dependent coefficient $a^{\prime}$ in Eq. (11). However, such a procedure yields terms proportional to $\phi_{1}$ in some response functions which do not vanish far away from the critical point, where the critical part should not play a role anymore. In principle, this problem can be solved by including crossover from singular critical behavior asymptotically close

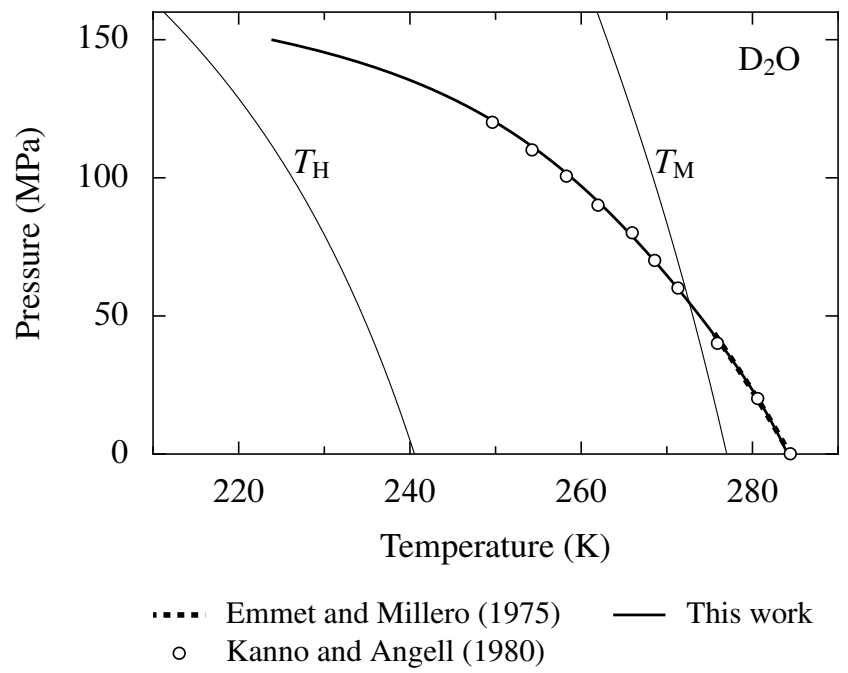

FIG. 18. Temperature of maximum density of $\mathrm{D}_{2} \mathrm{O}$ as a function of pressure according to the model (thick solid curve). $T_{\mathrm{M}}$ marks the melting curve ${ }^{64}$ and $T_{\mathrm{H}}$ denotes the homogeneous nucleation limit. ${ }^{68}$ Symbols represent experimental data. ${ }^{66,67}$

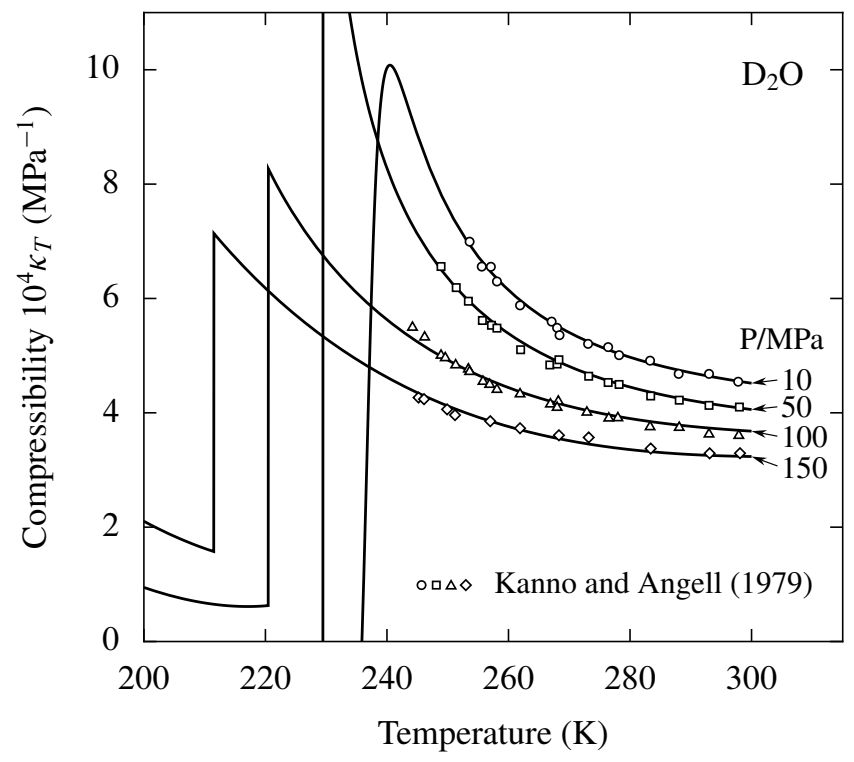

FIG. 19. Isothermal compressibility of $\mathrm{D}_{2} \mathrm{O}$ according to the model (curves). Symbols represent experimental data of Kanno and Angell. ${ }^{28}$

to the critical point to analytic behavior far away from the critical point, as has been done in the equation of state for $\mathrm{H}_{2} \mathrm{O}$ near the vapor-liquid critical point, ${ }^{103}$ and has also been suggested by Kiselev. ${ }^{5,6}$ However, it turns out that the theoretical model can represent all experimental data for $\mathrm{H}_{2} \mathrm{O}$ up to the maximum available pressure of $400 \mathrm{MPa}$, if we simply remove any constraints on the slope of the LLT and on the critical parameters in the unstable region which cannot be measured experimentally. With the addition of only two background terms, we were then able to fit almost all experimental data for supercooled water with this semi-empirical extension of the theoretical model. 


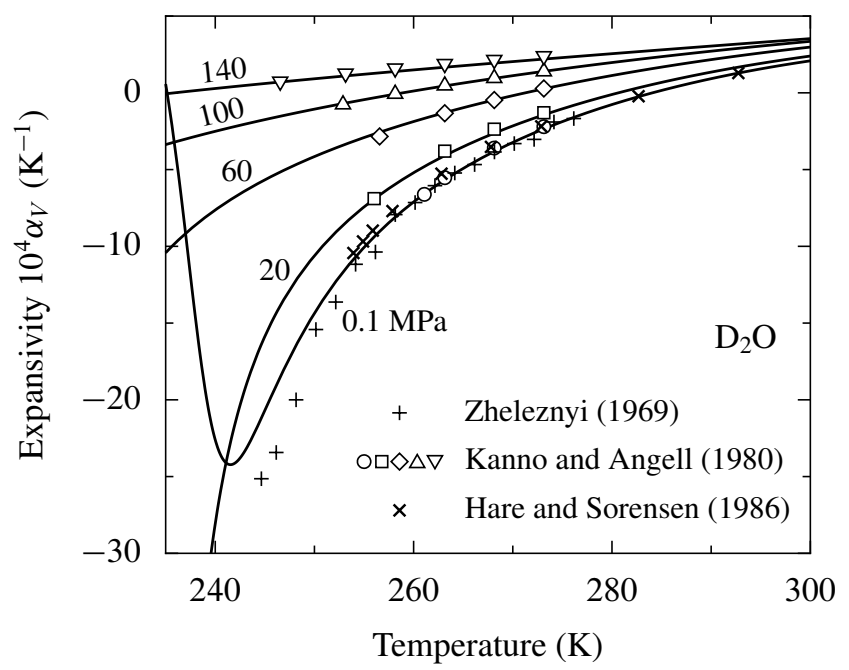

FIG. 20. Expansivity coefficient of $\mathrm{D}_{2} \mathrm{O}$ according to the model (curves). Symbols represent experimental data. ${ }^{29,65,67}$ The data of Zheleznyi ${ }^{65}$ (plusses) likely overestimate the anomaly in the expansivity (see the text).

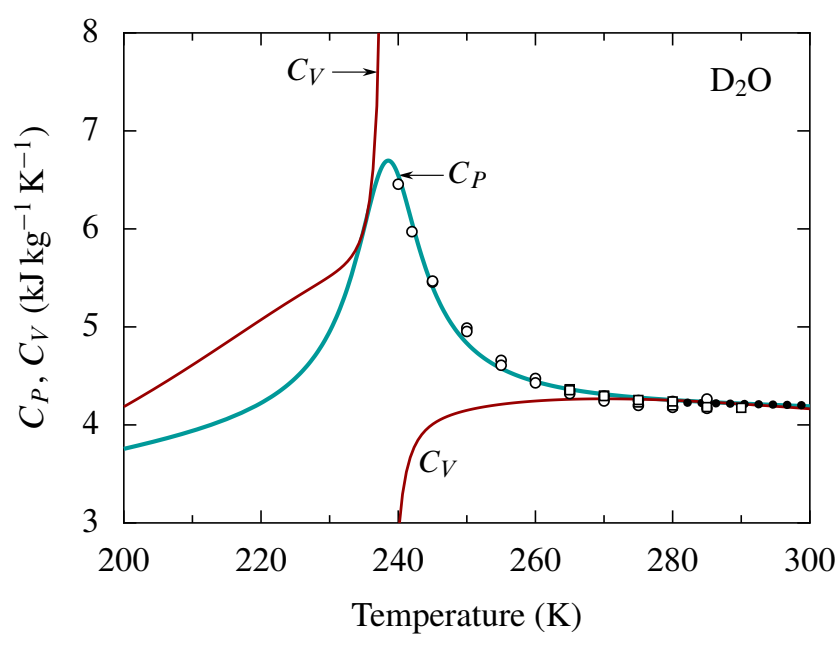

口 ○ Angell et al. (1982) .... Zábranský et al. (2010)

FIG. 21. Isobaric and isochoric heat capacity of $\mathrm{D}_{2} \mathrm{O}$ versus temperature at $0.1 \mathrm{MPa}$ according to the model (solid curves). Symbols represent experimental $C_{P}$ data of Angell et al. ${ }^{33}$ (squares: bulk water, circles: emulsion) and the dotted curve shows the correlation of Zábranský et al. ${ }^{98}$ for $C_{P}$. The predicted thermodynamic behavior of $C_{V}$ is discussed in Sec. VIC.

After fitting the model to a selection of experimental data ${ }^{17}$ at pressures up to $400 \mathrm{MPa}$, the parameters listed in Table III were obtained. Some parameters are significantly different from the earlier values. In particular, the critical pressure is about a factor of two higher than in the previous section, and the critical temperature is $10 \mathrm{~K}$ lower. Nevertheless, the extended model does represent almost all available experimental thermodynamic data for supercooled water. A comparison between the density values predicted by the model is presented in Fig. 23. The model reproduces the data, except for Mishima's points between $160 \mathrm{MPa}$ and $300 \mathrm{MPa}$

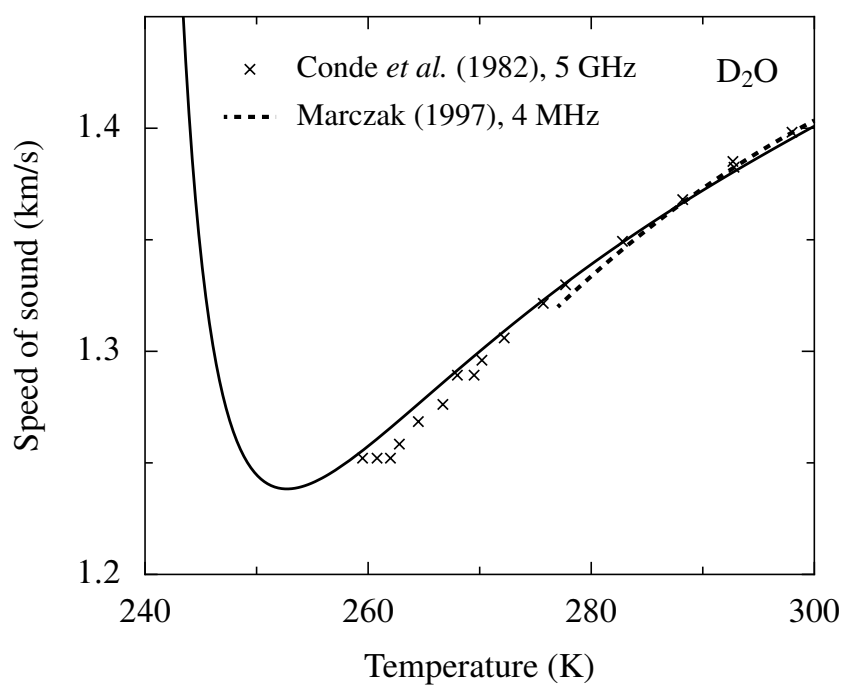

FIG. 22. Speed of sound of $\mathrm{D}_{2} \mathrm{O}$ at $0.1 \mathrm{MPa}$ according to the model (solid curve). Symbols represent experimental data of Conde et al. ${ }^{56}$ and the dashed line shows the correlation of Marczak. ${ }^{101}$

TABLE III. Parameter values for the extended model of $\mathrm{H}_{2} \mathrm{O}$

\begin{tabular}{lclc}
\hline \hline Parameter & \multicolumn{1}{c}{ Value } & Parameter & Value \\
\hline$T_{\mathrm{c}} / \mathrm{K}$ & 213.89 & $c_{12}$ & $-4.6569 \times 10^{-3}$ \\
$P_{\mathrm{c}} / \mathrm{MPa}$ & 56.989 & $c_{13}$ & $2.3627 \times 10^{-3}$ \\
$\rho_{\mathrm{c}} /\left(\mathrm{kg} \mathrm{m}^{-3}\right)$ & 949.87 & $c_{14}$ & $-2.8697 \times 10^{-4}$ \\
$a$ & 0.11624 & $c_{20}$ & $-3.6144 \times 10^{0}$ \\
$k$ & 0.43280 & $c_{21}$ & $-1.5009 \times 10^{-2}$ \\
$a^{\prime}$ & 0.10898 & $c_{22}$ & $-2.4609 \times 10^{-2}$ \\
$c_{02}$ & $4.0793 \times 10^{-2}$ & $c_{23}$ & $9.8679 \times 10^{-4}$ \\
$c_{03}$ & $-6.7912 \times 10^{-4}$ & $c_{30}$ & $5.4267 \times 10^{-1}$ \\
$c_{04}$ & $-7.5669 \times 10^{-6}$ & $c_{31}$ & $1.0620 \times 10^{-1}$ \\
$c_{05}$ & $1.0922 \times 10^{-5}$ & $c_{32}$ & $1.2759 \times 10^{-2}$ \\
$c_{11}$ & $1.9547 \times 10^{-1}$ & $c_{41}$ & $-7.9970 \times 10^{-2}$ \\
\hline \hline
\end{tabular}

below $230 \mathrm{~K}$. The temperature of maximum density is plotted in Fig. 12. The calculated behavior of the extended model is similar to that of the asymptotic model in the previous section in the range from $0 \mathrm{MPa}$ to $120 \mathrm{MPa}$. At higher pressures, the extended model predicts higher temperatures of maximum density than the previous model, but the results are still within the experimental uncertainty.

Compressibility data are compared with values of the extended model in Fig. 24. As in the previous model, the isobars of $0.1 \mathrm{MPa}$ and $10 \mathrm{MPa}$ intersect, but the intersection is located at a lower temperature. The data of Speedy and Angell ${ }^{27}$ and Kanno and Angell ${ }^{28}$ are well represented. The extended model also reproduces most of Mishima's data, which have a lower accuracy than the data of Angell and coworkers. Figure 25 shows the expansivity coefficient predicted by the extended model, which agrees with the data of Ter Minassian et $a l .{ }^{26}$ Below $250 \mathrm{~K}$, the model agrees better with the data of Hare and Sorensen of 1986 than with their data of 1987.

The calculated heat capacity at $0.1 \mathrm{MPa}$ is compared with experimental data in Fig. 26. For the extended model, the maximum of the heat capacity is lower than for the asymptotic 


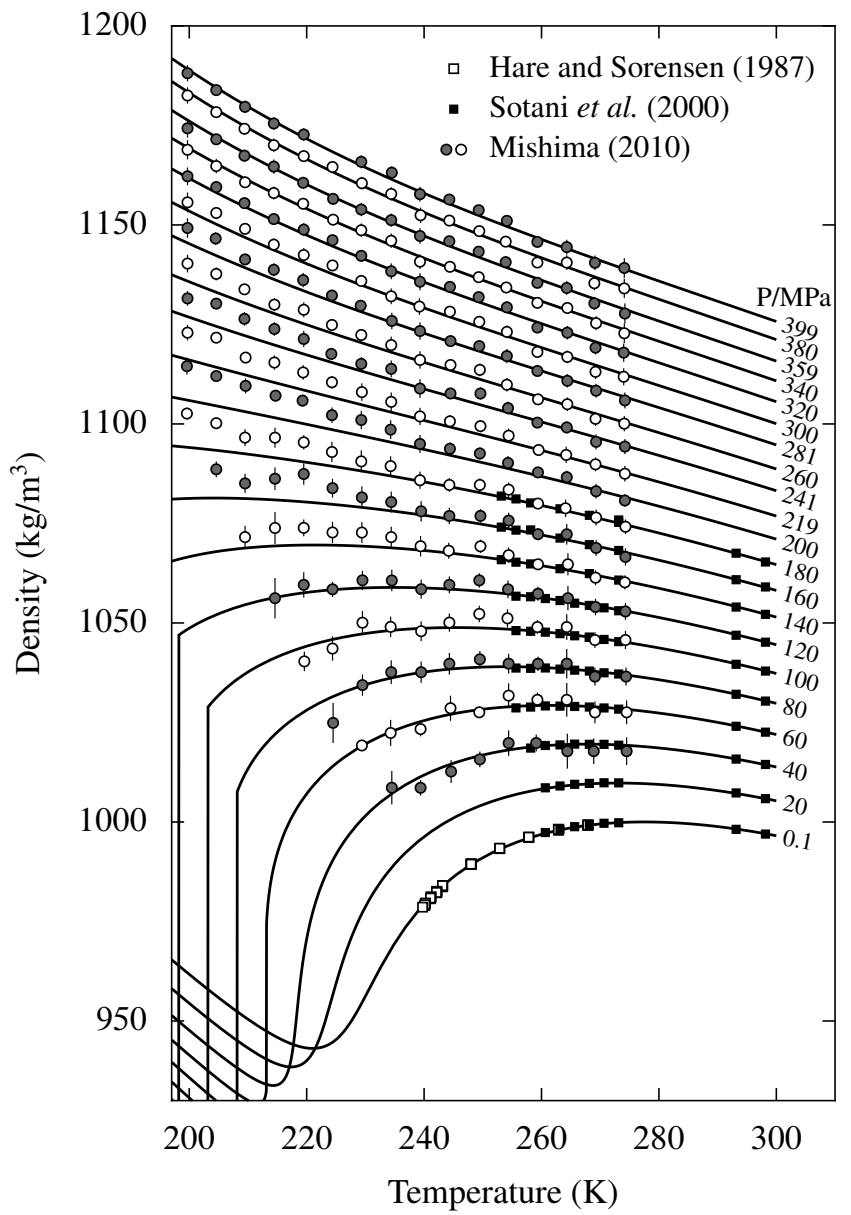

FIG. 23. Densities of $\mathrm{H}_{2} \mathrm{O}$ according to the extended model (curves). The symbols represent experimental data of Mishima, ${ }^{21}$ Sotani et $a l .{ }^{19}$ and Hare and Sorensen. ${ }^{18}$ The symbols for Mishima's densities on different isobars are alternatingly open and filled to guide the eye. The vertical lines through Mishima's points are uncertainties given by Mishima.

model, and the curvature of the data of Archer and Carter ${ }^{34}$ is better represented. The predicted heat capacity is shown as a function of pressure in Fig. 27. There are large differences between the results of the model and those of IAPWS95; the model predicts a minimum in the $250 \mathrm{~K}$ heat-capacity isotherm at about $240 \mathrm{MPa}$. A minimum in the heat capacity at this location was also predicted by Ter Minassian et al. ${ }^{26}$ based on their measurements of the expansivity coefficient.

The speed of sound predicted by the model, shown in Fig. 7, agrees fairly well with the experimental data of Taschin et $a l .47$

\section{PHYSICAL INTERPRETATION OF THE MODEL}

\section{A. Two states in supercooled water}

Two features make the second critical point in water phenomenologically different from the well-known vapor-liquid critical point. The negative slope of the liquid-liquid phase

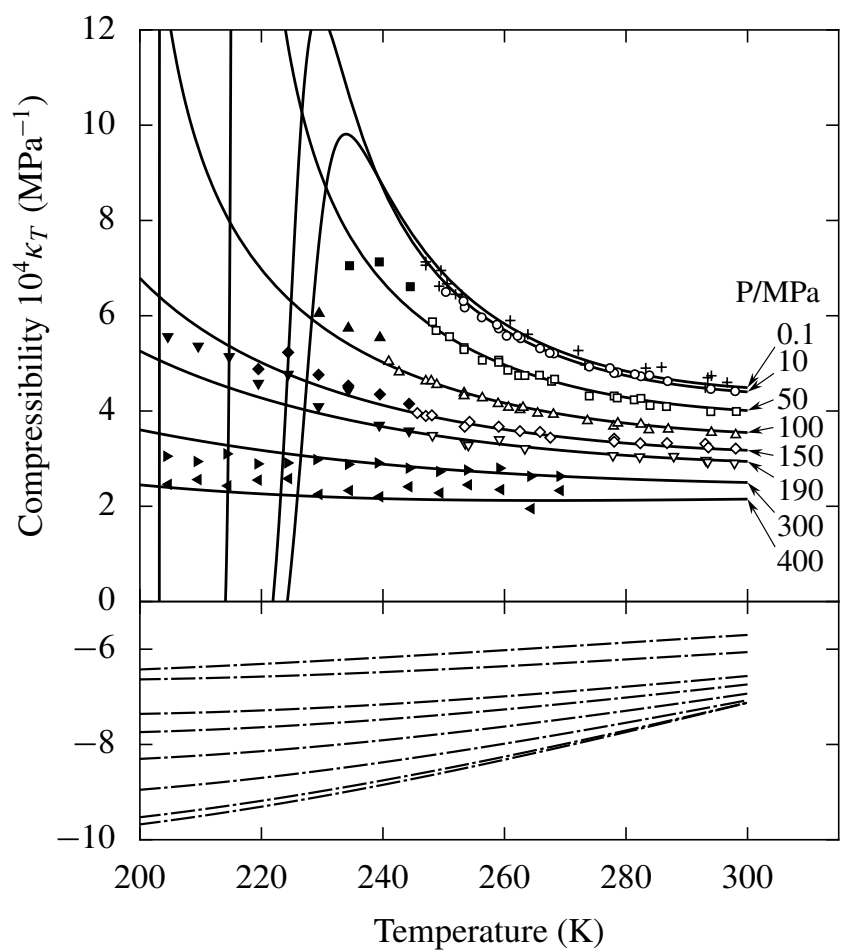

$\begin{array}{cl}+ & \text { Speedy and Angell (1976) } \\ \circ \square \Delta \diamond \nabla & \text { Kanno and Angell (1979) }\end{array}$

FIG. 24. Isothermal compressibility of $\mathrm{H}_{2} \mathrm{O}$ according to the extended model (curves). Symbols represent experimental data of Speedy and Angell, ${ }^{27}$ Kanno and Angell, ${ }^{28}$ and Mishima. ${ }^{21}$ Solid and open symbols with the same shape correspond to the same pressure. The regular backgrounds for the compressibility are shown by dash-dotted lines. The negative values of the regular parts are due to incorporating the fluctuation-induced critical background in $\chi_{2}$ as noted in Appendix A.

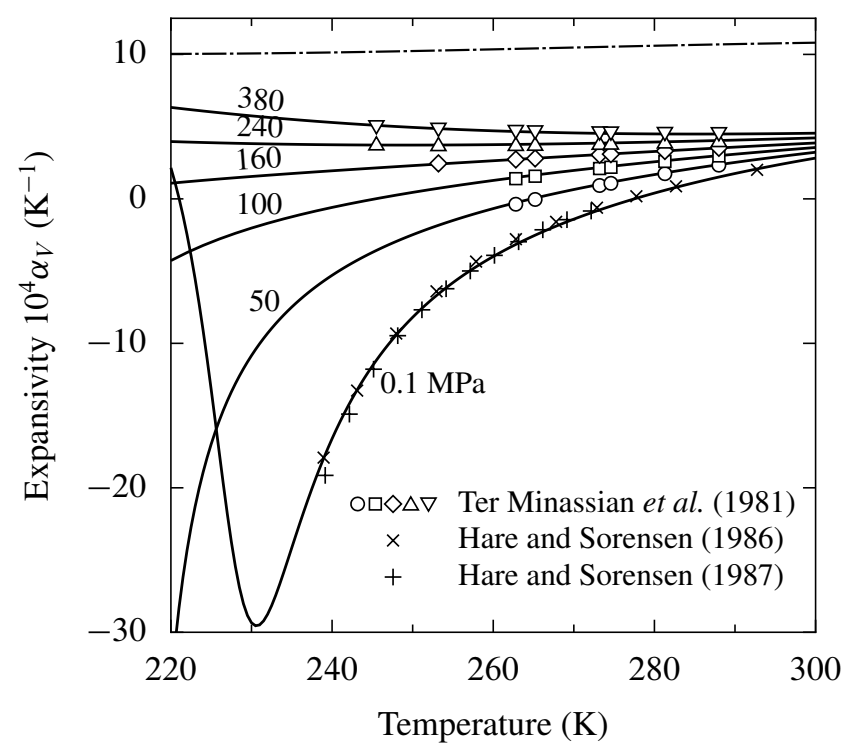

FIG. 25. Expansivity coefficient of $\mathrm{H}_{2} \mathrm{O}$ to the extended model (curves). Symbols represent experimental data of Ter Minassian et $a l^{26}$ and Hare and Sorensen. ${ }^{18,29}$ The dash-dotted line is the estimated regular part at $0.1 \mathrm{MPa}$. 


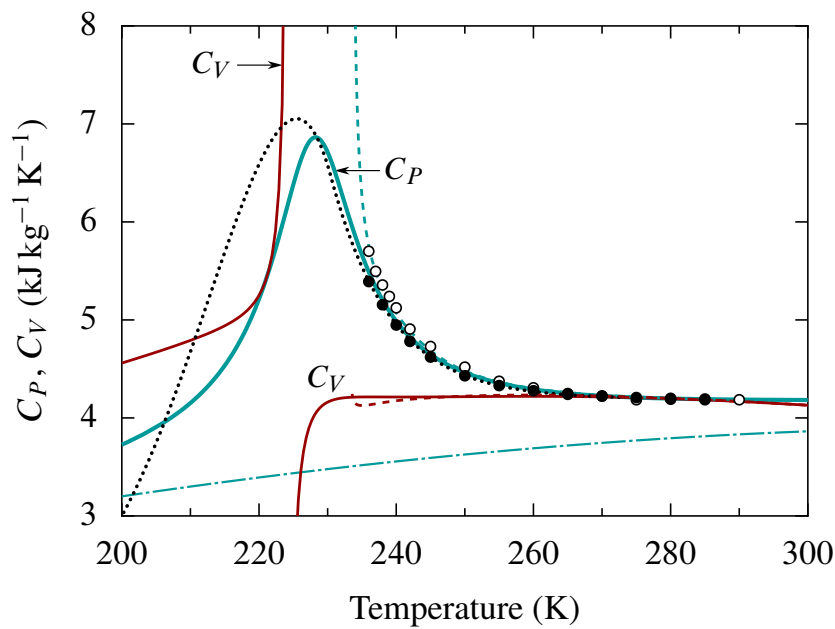

- Angell et al. (1982) - Archer and Carter (2000)

FIG. 26. Isobaric and isochoric heat capacity of $\mathrm{H}_{2} \mathrm{O}$ versus temperature at $0.1 \mathrm{MPa}$ according to the extended model (solid curves), IAPWS-95 (dashed), and the prediction of Murphy and Koop (dotted). Symbols represent experimental data of Angell et al. ${ }^{33}$ and Archer and Carter. ${ }^{34}$ The dash-dotted line is the estimated regular part of $C_{P}$. The predicted thermodynamic behavior of $C_{V}$ is discussed in Sec. VIC.

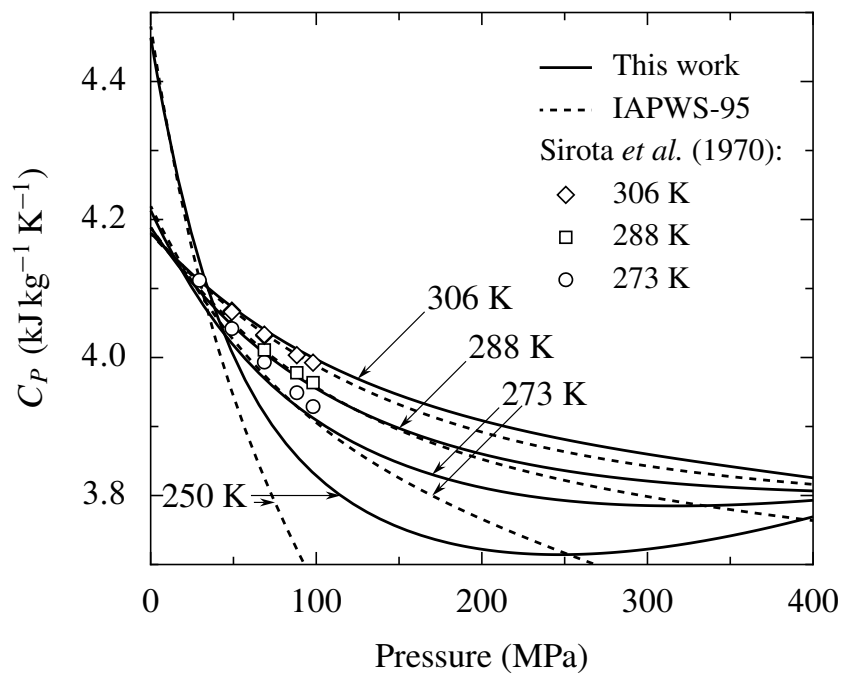

FIG. 27. Isobaric heat capacity of $\mathrm{H}_{2} \mathrm{O}$ versus pressure according to the extended model (solid curves) and IAPWS-95 (dashed). Symbols represent experimental data of Sirota et al. ${ }^{37}$

transition line in the $P-T$ plane means that high-density liquid water is the phase with larger entropy. The relatively large value of this slope at the liquid-liquid critical point (about 25 times greater than for the vapor-liquid transition at the critical point) indicates the significance of the entropy change relative to the density change, and, correspondingly, the importance of the entropy fluctuations. These features suggest that liquid-liquid phase separation in water is mostly driven by entropy rather than by energy, thus supporting the "latticeliquid" choice for the scaling fields given by Eqs. (11)-(13) with $b^{\prime}=0$.
As the first step to understand a relation between water's polyamorphism and the behavior of cold aqueous solutions, Bertrand and Anisimov ${ }^{3}$ have introduced a mean-field "twostate" model that clarifies the nature of the phase separation in a polyamorphic single-component liquid. Two-fluid-states models trace their lineage back to a 19th century paper by Röntgen. ${ }^{104}$ Relatively recently, Ponyatovskiı̌ et al. ${ }^{105}$ and, more quantitatively, Moynihan ${ }^{106}$ have described the emergence of a LLCP in supercooled water as resulting from the effects of nonideality in a mixture of two "components," with their fraction being controlled by thermodynamic equilibrium. However, while Moynihan assumed a "regular-solution" type of nonideality, which implies an energy-driven phase separation, such as the vapor-liquid transition or the conventional liquid-liquid transition in binary solutions, we believe that a near "athermal-solution" type of nonideality is mainly responsible for the entropy-driven phase separation in metastable water near the LLCP.

It is assumed that liquid water is a "mixture" of two states, $\mathrm{A}$ and $\mathrm{B}$, of the same molecular species. For instance, these two states could represent two different arrangements of the hydrogen-bond network in water and correspond to the lowdensity and high-density states of water. The fraction of water molecules, involved in either structure, denoted $\phi$ for state A and $1-\phi$ for state $\mathrm{B}$, is controlled by thermodynamic equilibrium between these two structures. Unlike a binary fluid, the fraction $\phi$ is not an independent variable, but is determined as a function of pressure $P$ and temperature $T$ from the condition of thermodynamic equilibrium. The simplest "athermal solution model" 107 predicts a symmetric liquid-liquid phase separation for any temperature, with the critical fraction $\phi_{\mathrm{c}}=1 / 2$, if the interaction parameter, which controls the excess (non-ideal) entropy of mixing is higher than its critical value. However, unlike a purely athermal non-ideal binary fluid, the entropy-driven phase separation in a polyamorphic single-component liquid will not be present at any temperature. On the contrary, the critical temperature $T_{\mathrm{c}}$ is to be specified through the temperature dependence of the equilibrium constant $K$ by

$$
\ln K=\lambda\left(\frac{1}{T}-\frac{1}{T_{\mathrm{c}}}\right),
$$

where $\lambda$ is the heat (enthalpy change) of "reaction" between $\mathrm{A}$ and B. A finite slope of liquid-liquid coexistence in the $P$ $T$ plane can be incorporated into the two-state lattice liquid model if one assumes that the Gibbs energy change of the "reaction" also depends on pressure.

A conceptually similar two-state model suggested by Hrubý and Holten ${ }^{108}$ has explained the inflection point in the surface tension (Fig. 6) by a rapid increase of the fraction of water molecules in the low-density state as water is cooled down.

\section{B. Liquid-liquid phase separation}

A consequence of the second critical point is a phase separation into a high-density and a low-density liquid at temperatures below the critical temperature. From the "lattice-liquid" 


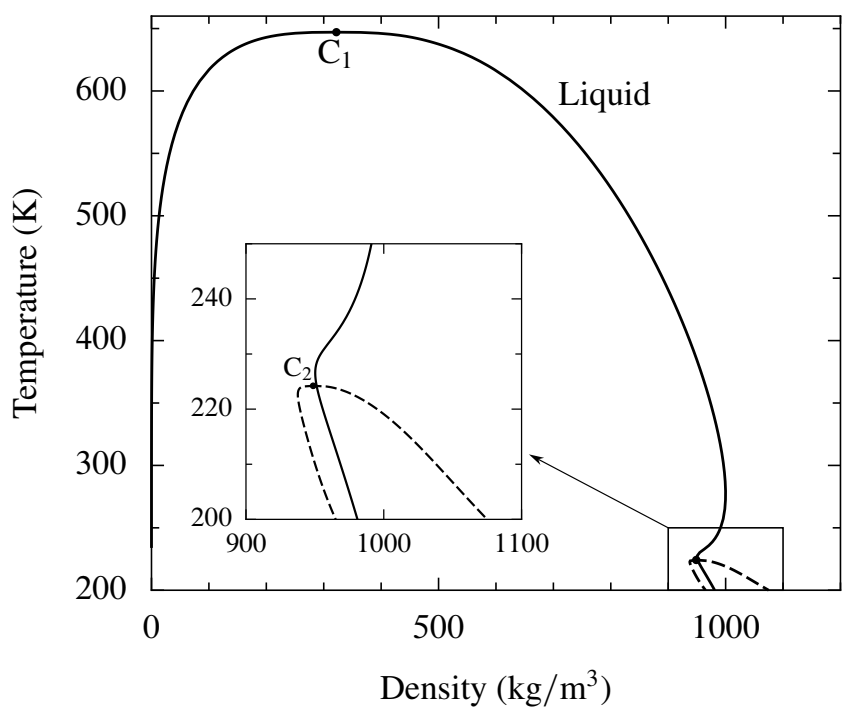

FIG. 28. Temperature-density diagram. $\mathrm{C}_{1}$ and $\mathrm{C}_{2}$ indicate the first and second critical point. The line left of $\mathrm{C}_{1}$ is the saturated vapor density according to IAPWS-95. Down to $250 \mathrm{~K}$, the line marked by 'Liquid' is the saturated liquid density computed with IAPWS95; below $250 \mathrm{~K}$, the line represents the liquid density at $0.1 \mathrm{MPa}$ predicted by our model. The dashed lines show the densities of the two liquid phases in equilibrium on the LLT line.

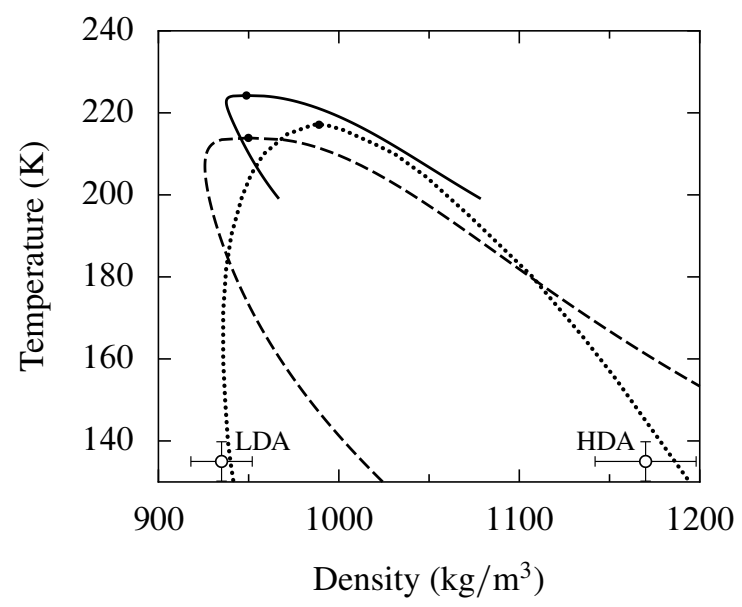

FIG. 29. Temperature-density diagram of the two liquid phases in equilibrium on the LLT line. Solid line: asymptotic model, dashed line: extended model, dotted line: prediction of the two-state regularsolution model of Moynihan. ${ }^{106}$ Solid dots mark the second critical points. Open circles are the densities of the low-density amorphous (LDA) and high-density amorphous (HDA) phases of water estimated from the experiments of Mishima. ${ }^{109}$

two-state model with a steep slope of the liquid-liquid transition (LLT) line in the $P-T$ phase diagram it follows that the phase separation is mostly driven by entropy rather than density. The LLT line is not exactly vertical in the phase diagram due to a small density difference between the two liquid phases, as pointed out by Bertrand and Anisimov. ${ }^{3}$ In Fig. 28, a temperature-density phase diagram is shown including both the vapor-liquid and liquid-liquid critical points. The region

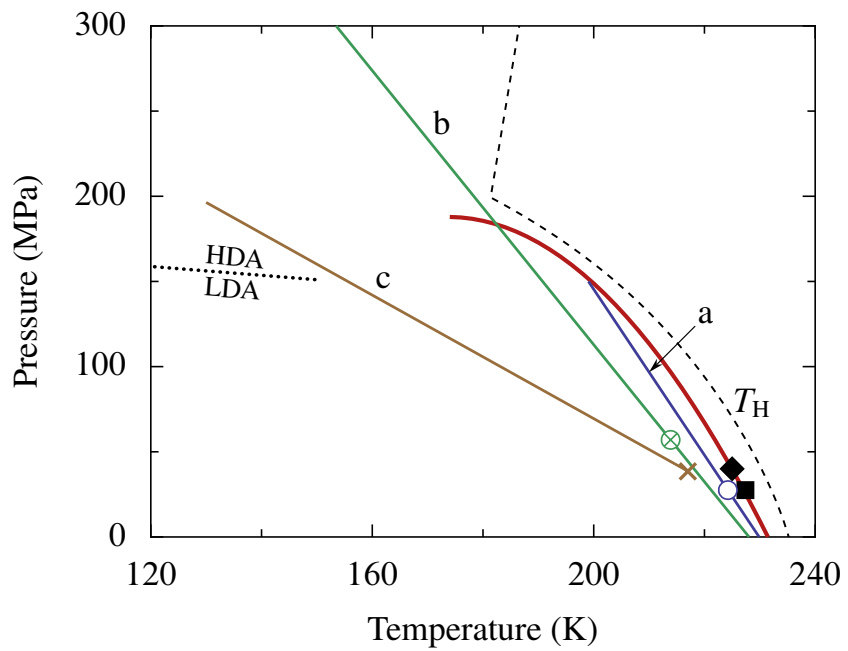

FIG. 30. Different linearizations of the LLT curve. The thick solid curve is the LLT curve suggested by Mishima. ${ }^{21}$ The lines marked $a$ and $b$ are the linearized LLT lines of our models restricted to $150 \mathrm{MPa}$ and $400 \mathrm{MPa}$, respectively; the line marked $\mathrm{c}$ is the LLT line of Moynihan. ${ }^{106}$ Symbols mark the liquid-liquid critical points; the solid diamond is the critical point of Mishima, ${ }^{21}$ the solid square is that of Bertrand and Anisimov, ${ }^{3}$ the open circle is that of our asymptotic model, the crossed circle is that of our extended model, and the cross is that of Moynihan. ${ }^{106}$ The dotted line is the phase-transition line between the two amorphous phases LDA and HDA as estimated by Whalley et al. ${ }^{110}$

below the second critical point is shown in more detail in Fig. 29, where predictions by several models of the densities of the two liquid phases are compared.

In the second-critical-point scenario it is assumed that the LLT line is connected to the phase-transition line between two amorphous phases found below about $130 \mathrm{~K} .{ }^{111}$ Since the amorphous phase-transition line is nearly horizontal in the $P-T$ diagram, as shown in Fig. 30, it follows that the LLT must be strongly curved to account for such a connection. In our model, we have linearized the LLT, so the predicted equilibrium densities at $135 \mathrm{~K}$ differ from the experimental densities of the amorphous phases, as seen in Fig. 29. The two-state model of Moynihan ${ }^{106}$ was fitted to the experimental amorphous-phase densities, as shown in Fig. 29. In Moynihan's model, the LLT is also a straight line, but its slope, $\mathrm{d} P / \mathrm{d} T$, is less than half of the slope of our LLT lines (Fig. 30). Comparing our models for the 0-150 MPa and 0-400 MPa ranges, we see that a lower LLT slope is needed for the larger pressure range. In conclusion, with a linearized LLT, the larger the desired temperature and pressure range of the model, the smaller $\mathrm{d} P / \mathrm{d} T$ slope of the linearized LLT line is required.

Based on the fit of the model to the experimental data, the liquid-liquid critical point can be located in a certain range of temperatures and pressures, depending on the pressure limit for experimental data involved in the fit. Without any preliminary idea on the location of the LLT curve, the position of the liquid-liquid critical point is uncertain. In principle, we cannot exclude even a negative value for the critical pressure. Our assumption on the LLT curve is based on the predic- 

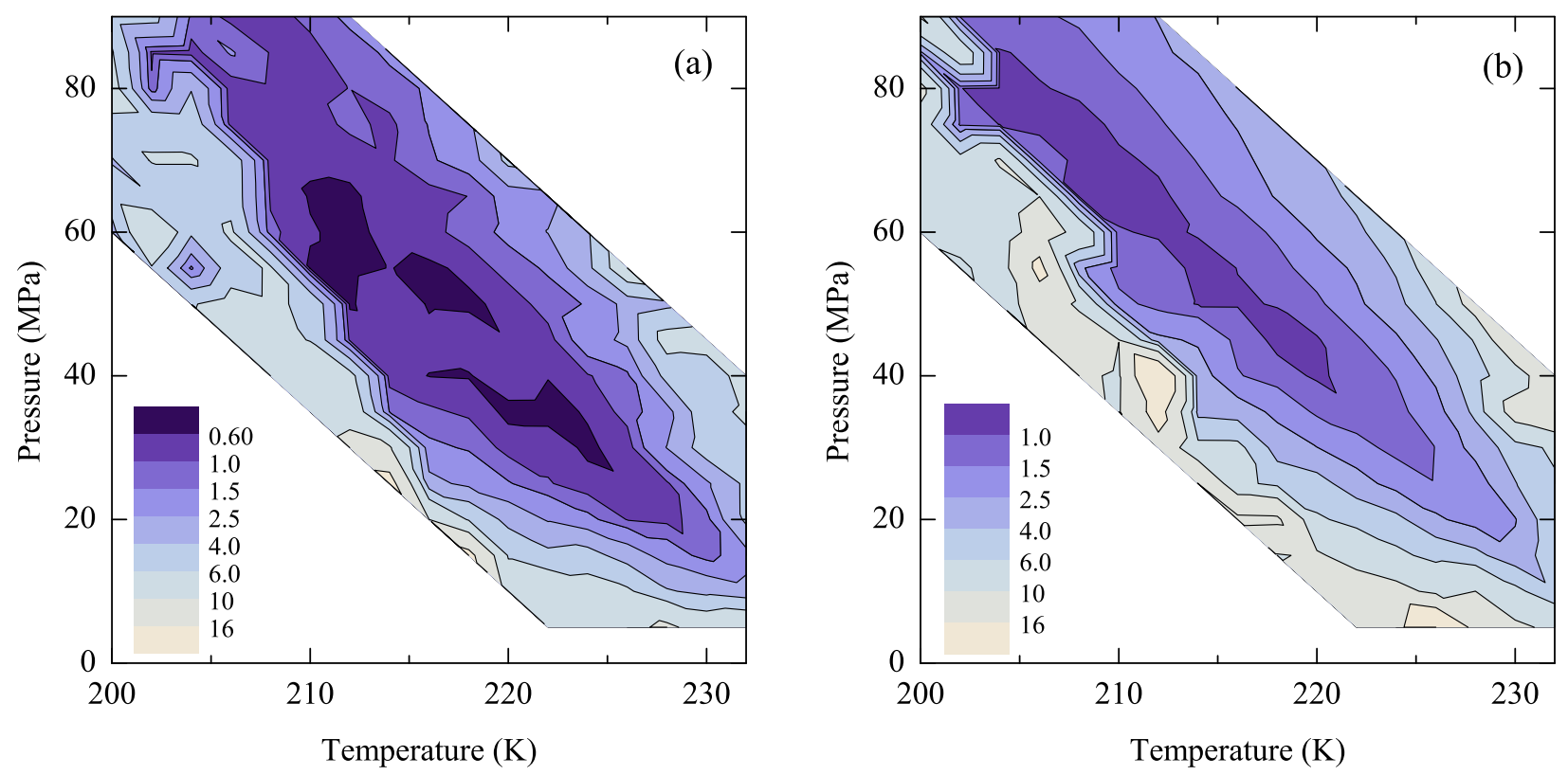

FIG. 31. Reduced sum of squared residuals as a function of the location of the liquid-liquid critical point for $\mathrm{H}_{2} \mathrm{O}$. (a) Asymptotic model, fitted to experimental data up to $150 \mathrm{MPa}$; (b) Extended model, fitted to experimental data up to $400 \mathrm{MPa}$.

tions of different authors ${ }^{21,28,62,112}$ which give approximately the same result. We have linearized this curve for a certain range of pressures. If the data are restricted up to $100 \mathrm{MPa}$, the critical pressure is optimized to $28 \mathrm{MPa}$ and the critical temperature is found to be $227 \mathrm{~K}^{3}$ If we include more high-pressure data and linearize the LLT curve in the pressure range up to $150 \mathrm{MPa}$, the optimal value for the critical pressure becomes less certain and moves up. This tendency continues when we include high-pressure data and linearize the LLT curve up to $400 \mathrm{MPa}$. As illustrated in Fig. 31(a), best fits for the $150 \mathrm{MPa}$ model are obtained with critical-pressure values between about $25 \mathrm{MPa}$ and $70 \mathrm{MPa}$. For the extended, $400 \mathrm{MPa}$, model, the optimum critical-pressure values move to a higher range from $40 \mathrm{MPa}$ to $85 \mathrm{MPa}$ [Fig. 31(b)].

\section{Absolute stability limit of the liquid state}

For the supercooled liquid state to be thermally and mechanically stable, both the isochoric heat capacity $C_{V}$ and the isothermal compressibility $\kappa_{T}$ must be positive. The scaling model predicts regions in the phase diagram where $C_{V}$ or $\kappa_{T}$ is negative, as shown in Fig. 32. As noted by Bertrand and Anisimov, ${ }^{3}$ the stability locus is located close to the Widom line, but its exact location depends on the parameters of the model. Our model predicts that the thermal stability condition is violated before the mechanical stability condition as water is cooled down, contrary to the violation order shown by a cubic equation of state that describes the vapor-liquid transition. ${ }^{113,114}$ At pressures slightly above the critical pressure, the mechanical stability limit coincides with the LLT line; that is, one of the liquid phases (the low-density liquid) is unstable. We note that a spinodal for the LLT is not defined in the scaled equation of state. ${ }^{115}$

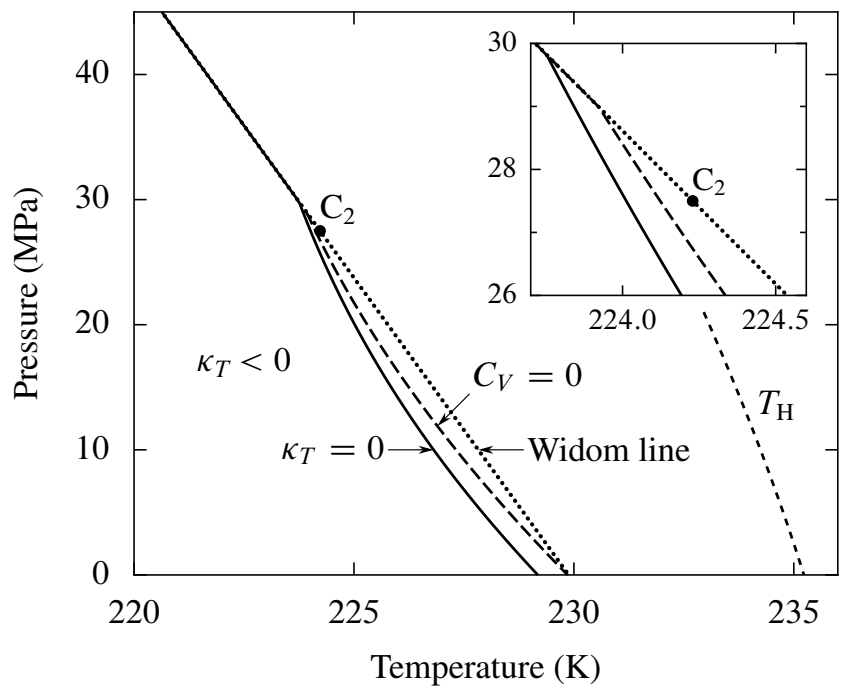

FIG. 32. Absolute stability limit of the liquid state, predicted by the model. Solid line: limit of mechanical stability, where the isothermal compressibility $\kappa_{T}$ is zero. Long dashed line: limit of thermal stability, where the isochoric heat capacity $C_{V}$ is zero. $T_{\mathrm{H}}$ marks the experimental homogeneous nucleation limit, and $\mathrm{C}_{2}$ indicates the second critical point. The dotted line, at which $h_{1}=0$ [Eq. (11)], indicates the liquid-liquid transition line and its extension below the critical pressure, the Widom line. The inset shows the stability limits in the vicinity of the critical point.

The isochoric heat capacity $C_{V}$ is related to the isobaric heat capacity by ${ }^{1}$

$$
C_{V}=C_{P}-\frac{T \alpha_{V}^{2}}{\rho \kappa_{T}}
$$




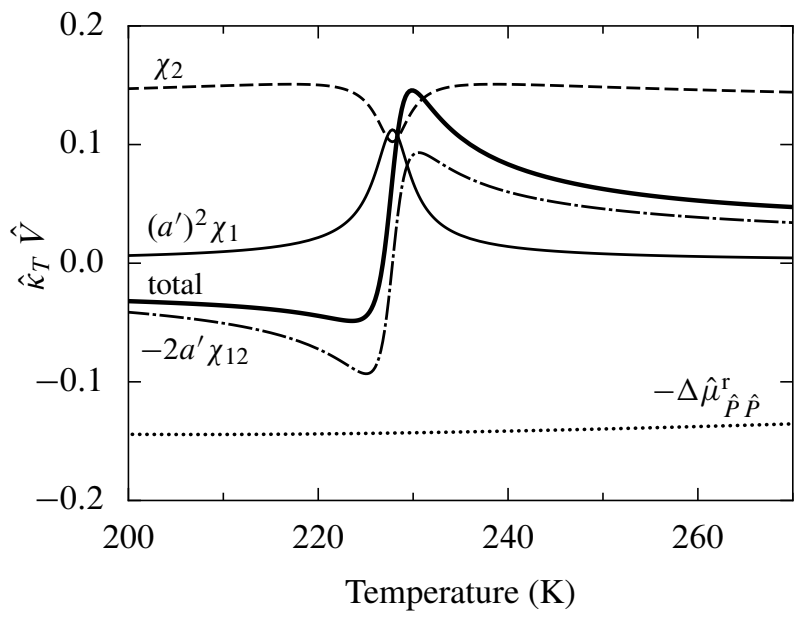

FIG. 33. Contribution of the susceptibilities, $\chi_{1}, \chi_{2}$, and $\chi_{12}$, and the regular part to the compressibility, as given by Eq. (18), at $10 \mathrm{MPa}$. The thick line represents the total compressibility multiplied by the volume, $\hat{\kappa}_{T} \hat{V}$.

The critical part of $C_{V}$ expressed through the scaling susceptibilities is derived in Appendix B. As the compressibility $\kappa_{T}$ becomes smaller with decreasing temperature, $C_{V}$ first becomes negative and then diverges to negative infinity as $\kappa_{T}$ approaches zero. At temperatures below the mechanical stability limit, where $\kappa_{T}$ is negative, $C_{V}$ is larger than $C_{P}$ (Fig. 15). Another property that diverges is the speed of sound $c$, which is related to $C_{P}, C_{V}$ and $\kappa_{T}$ according to ${ }^{1}$

$$
c=\left(\rho \kappa_{T} C_{V} / C_{P}\right)^{-1 / 2} .
$$

The speed of sound diverges to positive infinity (Fig. 7) when $C_{V}$ approaches zero (at the thermal stability limit), which occurs at a slightly higher temperature than the zerocompressibility limit. In the unstable region, the speed of sound is imaginary.

Kiselev ${ }^{5}$ has computed a kinetic spinodal of supercooled water and found that this stability limit was located a few kelvin below the homogeneous nucleation limit. Kiselev calls the area in the phase diagram below the stability limit a "nonthermodynamic habitat," not because liquid water is necessarily mechanically unstable there, but because the lifetime of liquid water is smaller than the time needed for equilibration. Using molecular simulations, Moore and Molinero ${ }^{116}$ have also found a stability limit just below the homogeneous nucleation temperature. They show that below this limit, the time for crystallization is shorter than the relaxation time of the liquid, so liquid water cannot not be equilibrated.

An important question arises: What terms in our model are responsible for the instability of the liquid state? Figure 33 shows the contributions of the individual susceptibilities to the compressibility, as given by Eq. (18). The strong and weak susceptibilities $\chi_{1}$ and $\chi_{2}$ are positive in the entire temperature range, whereas the cross susceptibility $\chi_{12}$ changes sign on the Widom line. The sum of the contributions of the three susceptibilities is positive in the entire range; it is the contribution of the regular part, which is negative, that results in a negative compressibility below a certain temperature.

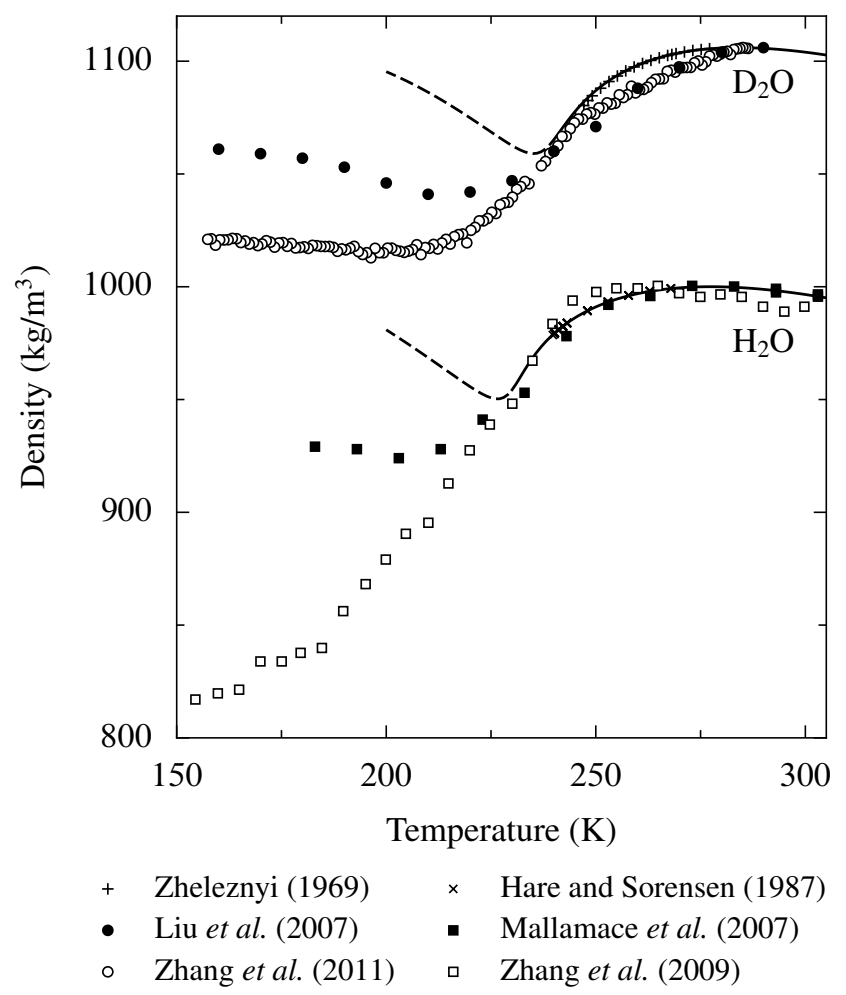

FIG. 34. Experimental densities of $\mathrm{D}_{2} \mathrm{O}$ (open and filled circles, both in hydrophilic confinement), ${ }^{112,117}$ and $\mathrm{H}_{2} \mathrm{O}$ (open squares: hydrophobic confinement, filled squares: hydrophilic confinement), ${ }^{118,119}$ together with bulk densities ${ }^{18,65}$ (plus and cross symbols). The predictions of the asymptotic scaling models for bulk $\mathrm{D}_{2} \mathrm{O}$ and $\mathrm{H}_{2} \mathrm{O}$ are shown by solid curves, and the dashed curves represent the extrapolation in the unstable region.

\section{Confined water}

New insights into the thermodynamics of supercooled water have been provided by measurements of water confined in mesoporous structures. The silica material MCM-41, which is comprised of a "honeycomb" of long cylindrical nanopores, has received significant attention as a confining medium in part because the diameter of the pores can be precisely controlled. As the pore diameter is decreased, the freezing point of water is reduced. Spontaneous crystallization is completely suppressed below a critical pore diameter, which allows for measurements to be made below the homogeneous-nucleation temperature. Examples of such measurements for hydrated MCM-41 are presented for the density in Fig. 34, for the expansion coefficient in Fig. 35, and for the heat capacity in Fig. 36, to be compared with the prediction for bulk water.

Many of the results of experiments on confined water can be interpreted in the framework of the second critical point hypothesis. Remarkably, the heat capacities exhibit maxima upon crossing the Widom line predicted for bulk water. However, the height of the maximum and the shape of the heat capacity in porous media differ significantly from those predicted in bulk water (Fig. 36). It has recently been reported that the density of confined water shows significant hysteresis 


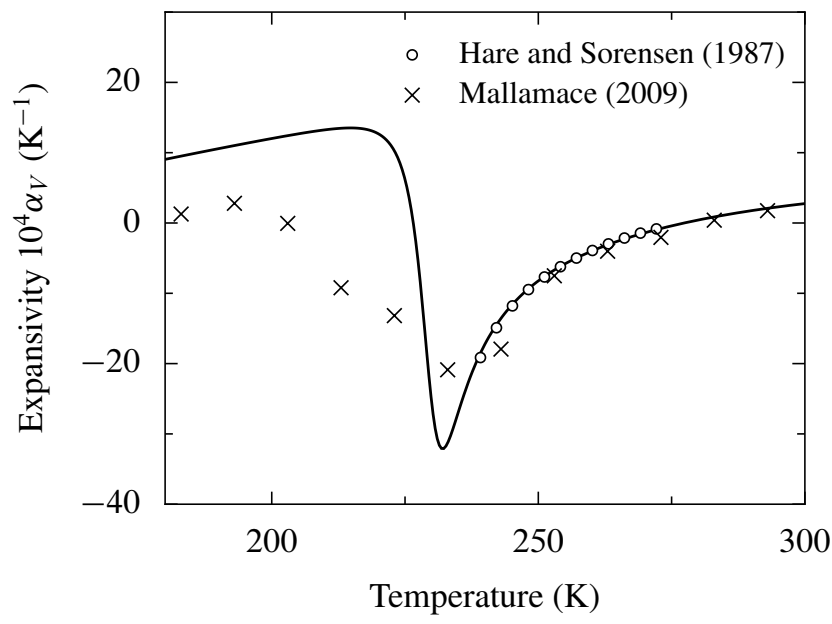

FIG. 35. Thermal expansion coefficient in confined water as measured by Mallamace (crosses). ${ }^{120}$ For comparison, the expansion coefficient of bulk water is also shown (circles). The curve is the prediction of the asymptotic model.

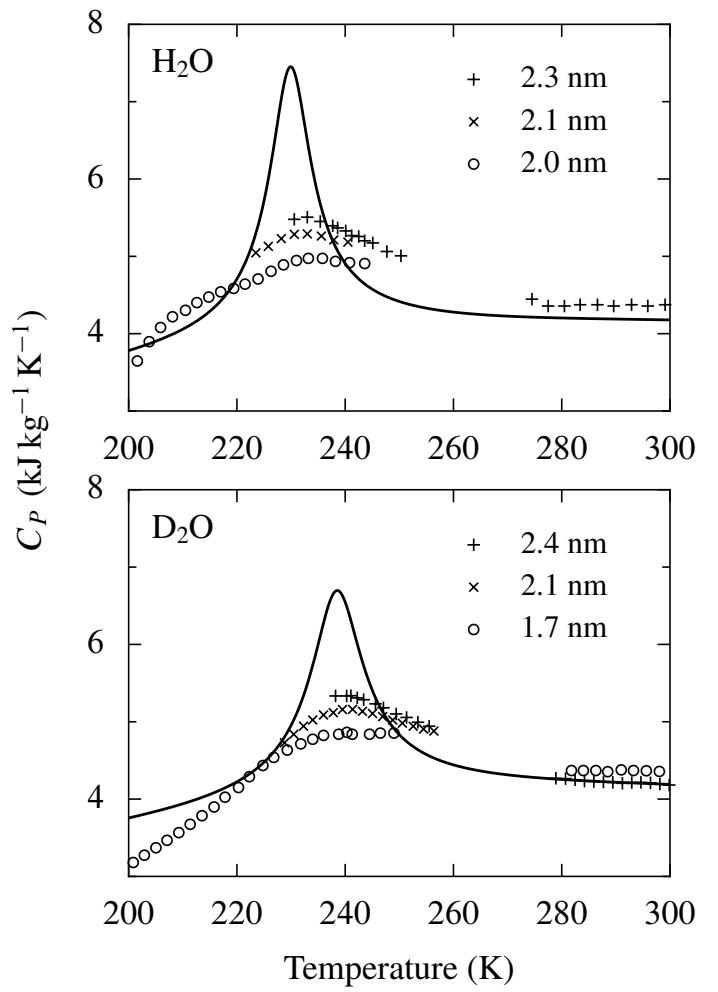

FIG. 36. Heat capacity of $\mathrm{H}_{2} \mathrm{O}$ and $\mathrm{D}_{2} \mathrm{O}$ in silica nanopores as measured by Nagoe et al. ${ }^{121}$ Different symbols correspond to different pore diameters. The curves are the predictions of the asymptotic model.

with temperature variation at elevated pressures. ${ }^{112}$ This can be interpreted in terms of the crossing of the first-order liquidliquid coexistence curve. The exact connection between the bulk thermodynamics of supercooled water and the results found in confinement is an active area of research. Surface effects, both in the form of finite-size effects and surface-water interactions, are thought to be contributing factors to the observed differences. In computer simulations of water confined in MCM-41, investigators have found that water found in the immediate vicinity of the surface behaves differently from water in the center of the pore. ${ }^{122}$ This distinction raises the possibility that wetting-type phenomena may be responsible for some of the observed behavior. That the surface interactions play a significant role can be seen from the fact that the measured density for hydrophobic and hydrophilic confinement are qualitatively different. ${ }^{119}$ However, even in similar hydrophilic environments, the measurements for $\mathrm{D}_{2} \mathrm{O}$ show considerable difference (Fig. 34). The difference could be caused by the uncertainty in the background of the neutron diffraction intensity, which must be subtracted from the measured intensity in neutron-scattering experiments. ${ }^{112,119}$ In addition, the relative densities that are obtained must be scaled to obtain absolute densities, which introduces an additional uncertainty. The shape of the response functions, the expansivity coefficient and the heat capacity, as shown in Figs. 35 and 36, is more certain. The rounded anomalies of the heat capacity around the Widom line in the confined $\mathrm{mW}$ water model are demonstrated by recent simulations of $\mathrm{Xu}$ and Molinero. ${ }^{123}$ An initial qualitative analysis of the finite-size effects based on the LLCP hypothesis has been made by Bertrand and Anisimov. ${ }^{3}$ This work indicates that finite-size effects can account for some of the differences between confined and bulk results.

\section{DISCUSSION}

We have evaluated the IAPWS-95 formulation for ordinary water when it is extrapolated into the supercooled region. At 0.1 MPa, it was found that IAPWS-95 reproduces the experimental data of all properties except the isobaric heat capacity. With increasing pressure, however, the deviations of the IAPWS-95 values from the data become larger and larger. A property that is particularly poorly described is the expansion coefficient, for which the IAPWS-95 extrapolation has a wrong sign in a large region of the phase diagram. Even in the stable region close to the melting line, where IAPWS95 should be valid, the expansion coefficient predicted by IAPWS-95 is a factor of two lower than the experimental value. The IAPWS equation for the surface tension performs well when extrapolated down to about $266 \mathrm{~K}$; below that, the experimentally observed increase of the temperature dependence is not reproduced and the difference between the equation and the data increases with decreasing temperature.

We have demonstrated that a theoretical model based on the assumption of a liquid-liquid critical point in supercooled water can represent the thermodynamic properties of both supercooled $\mathrm{H}_{2} \mathrm{O}$ and $\mathrm{D}_{2} \mathrm{O}$ to pressures of $150 \mathrm{MPa}$. Moreover, by allowing the slope of the liquid-liquid transition (LLT) line and the critical pressure to be freely adjustable parameters the model can represent almost all available thermodynamic property data for supercooled water. Nevertheless, there are still a number of issues that need to be considered.

A principal issue is that the existence of a liquid-liquid critical point is not the only possible explanation for the anoma- 
lous behavior of the thermodynamic properties of supercooled water. Scenarios for a singularity-free or critical-point-free interpretation have also been proposed. ${ }^{124-126}$ Recently, Stokely et al. ${ }^{127}$ have shown, for a water-like lattice model, how all such scenarios can be described by varying two quantities, the strength of the hydrogen bonds and the cooperativity of the hydrogen bonds. An intriguing possibility of the existence of multiple critical points in supercooled water, as predicted by some simulations, ${ }^{128,129}$ is discussed by Brovchenko and Oleinikova. ${ }^{130}$ Another possibility is that response functions like the compressibility do not diverge at a single temperature corresponding to a critical temperature but at a range of pressure-dependent temperatures $T_{\mathrm{S}}(P)$ corresponding to a crystallization spinodal, the absolute limit of stability of the liquid phase. Most recently, the discussion on the nature of the anomalies observed in supercooled water received an additional impetus after Limmer and Chandler reported new simulation results ${ }^{9}$ for two atomistic models of water, $\mathrm{mW}^{131}$ and mST2. ${ }^{132}$ They did not find two liquid states in the supercooled region and excluded the possibility of a critical point for the models studied. However, the most recent simulations by Sciortino et al. ${ }^{133}$ of the original ST2 model confirm the existence of a liquid-liquid critical point for that particular model, with two distinct liquid states. They found no evidence of crystallization during their simulation time, while there was enough time for the liquid to equilibrate. It would be important to compare the anomalies obtained for various models with those exhibited by real water.

Recent simulations of Moore and Molinero ${ }^{116}$ indicate that for simulation cells larger than the critical ice nucleus, spontaneous crystallization occurs before liquid-liquid separation can equilibrate. An approach to consider a coupling between spontaneous crystallization near the absolute stability limit of the liquid state and liquid-liquid separation is the theory of weak crystallization pioneered by Brazovskiı̌, ${ }^{134}$ further developed by Brazovskiı et al., ${ }^{135}$ and reviewed by Kats et al. ${ }^{136}$ According to this theory, the fluctuations of the translational (short-wavelength) order parameter $\psi$ renormalize the meanfield distance $\Delta_{0}=\left(T-T_{0}\right) / T$ between the temperature $T$ and the mean-field absolute stability limit $T_{0}$ of the liquid phase:

$$
\Delta=\Delta_{0}+\kappa \Delta^{-1 / 2},
$$

where $\Delta=\left(T-T_{\mathrm{s}}\right) / T$ where $T_{\mathrm{S}}$ is the fluctuation-affected stability-limit temperature, and $\kappa$ is a molecular parameter, similar to the Ginzburg number that defines the validity of the mean-field approximation in the theory of critical phenomena. ${ }^{82,137}$ The theory of weak crystallization requires $\kappa \Delta^{-1 / 2} \ll \Delta_{0}$ and $\Delta_{0} \ll 1$. There are two non-trivial consequences of the effects of translational-order fluctuations: (1) The heat capacity would contain a fluctuation correction $\delta C_{P} \sim \Delta^{-3 / 2}$; (2) The renormalized gap $\Delta$ may be positive even at negative $\Delta_{0}$, meaning that translational-order fluctuations stabilize the liquid phase.

The theory of weak crystallization was used to describe a coupling between one-dimensional density modulation and orientational fluctuations in liquid crystals; it was supported by accurate light-scattering and heat-capacity measurements. ${ }^{137-139}$
Similarly, we could consider a coupling between the translational-order fluctuations and the critical fluctuations of the liquid-liquid order parameter $\phi_{1}$. A relationship between the structural transformation in liquid water and its crystallization rate is suggested by recent simulations by Moore and Molinero. ${ }^{116}$ The lowest-order term associated with such a coupling in the free-energy Landau expansion would be $\sim \psi^{2} \phi_{1}$. Such a coupling would be possible even if the virtual critical point is below the stability limit of the liquid phase.

Formation of ice is not "weak crystallization." This is why application of weak crystallization theory to supercooled water is questionable. However, it may not be hopeless. The gap $\Delta$ between the melting temperature $T_{\mathrm{m}}$ and the temperature of spontaneous crystallization $T_{\mathrm{H}}$ is of the order of 0.1 . Hence, a further investigation of the possibility of such coupling would be worthwhile. The actual question is how the experimental observations can be explained by a theory that accounts for both crystallization and liquid-liquid separation. The final conclusion concerning the existence of the liquid-liquid critical point in water should be based on the ability to quantitatively describe the experimental data.

Assuming the existence of the LLCP in supercooled water, we confirm the finding of Fuentevilla and Anisimov ${ }^{7}$ and Bertrand and Anisimov ${ }^{3}$ that the critical point is located at much lower pressure than predicted by most simulations, definitely below $100 \mathrm{MPa}$, while the precise value of the critical pressure is uncertain. However, regardless of all open questions, we have shown that a critical-point parametric equation of state describes the available thermodynamic data for supercooled water within experimental accuracy, thus establishing a benchmark for any further developments in this area.

\section{ACKNOWLEDGMENTS}

We acknowledge collaboration with D. Fuentevilla and J. Kalová at an early stage of this research. We thank R. Torre for sending us the experimental data on the sound velocity, C. A. Angell, A. H. Harvey, S. Sastry, and H. E. Stanley for useful comments and suggestions, and S.-H. Chen and Y. Zhang for discussing their results on confined supercooled water. We also thank J. P. O'Connell for clarifying the independence of the mechanical and thermal instability criteria. The research of V. Holten at the University of Maryland has been supported by the Burgers Program of the University of Maryland (four months). The research of C. E. Bertrand and current research of V. Holten has been supported by the Division of Chemistry of the US National Science Foundation under Grant No. CHE-1012052. Travel support for V. Holten was provided by the J. M. Burgerscentrum in the Netherlands.

\section{Appendix A: Linear-model parametric equations}

The formulas for the linear model in this appendix are taken from Behnejad et al. ${ }^{81}$ [In Behnejad et al. ${ }^{81}$ the formula corresponding to our Eq. (A15) erroneously does not contain the factor $L_{0}$.] 
The $r$ and $\theta$ coordinates are related to $h_{1}$ and $h_{2}$ by

$$
\begin{aligned}
& h_{1}=a r^{\beta+\gamma} \theta\left(1-\theta^{2}\right), \\
& h_{2}=r\left(1-b^{2} \theta^{2}\right),
\end{aligned}
$$

with $\gamma=2-\alpha-2 \beta$ and

$$
b^{2}=\frac{\gamma-2 \beta}{\gamma(1-2 \beta)} .
$$

The scaling densities are given by

$$
\begin{aligned}
& \phi_{1}=k r^{\beta} \theta \\
& \phi_{2}=a k r^{1-\alpha} s(\theta),
\end{aligned}
$$

with

$$
\begin{aligned}
s(\theta) & =L_{0}\left(s_{0}+s_{2} \theta^{2}\right), \\
L_{0} & =1 /\left[2 b^{4}(1-\alpha) \alpha\right], \\
s_{0} & =(\gamma-2 \beta)-b^{2} \alpha \gamma, \\
s_{2} & =(\alpha-1)(\gamma-2 \beta) b^{2} .
\end{aligned}
$$

The scaling susceptibilities are given by

$$
\begin{aligned}
\chi_{1} & =\frac{k}{a} r^{-\gamma} q_{1}(\theta), \\
\chi_{12} & =k r^{\beta-1} q_{12}(\theta), \\
\chi_{2} & =a k r^{-\alpha} q_{2}(\theta),
\end{aligned}
$$

with

$$
\begin{aligned}
q_{1}(\theta)= & \left(1-b^{2} \theta^{2}+2 \beta b^{2} \theta^{2}\right) / q_{0}(\theta), \\
q_{12}(\theta)= & \theta\left[-\gamma+(\gamma-2 \beta) \theta^{2}\right] / q_{0}(\theta), \\
q_{2}(\theta)= & {\left[(1-\alpha)\left(1-3 \theta^{2}\right) s(\theta)\right.} \\
& \left.-(\beta+\gamma) 2 s_{2} L_{0} \theta^{2}\left(1-\theta^{2}\right)\right] / q_{0}(\theta), \\
q_{0}(\theta)= & \left(1-3 \theta^{2}\right)\left(1-b^{2} \theta^{2}\right)+2 b^{2}(\beta+\gamma) \theta^{2}\left(1-\theta^{2}\right) .
\end{aligned}
$$

The weakly divergent scaling susceptibility $\chi_{2}$ also contains a negative fluctuation-induced analytical background, which, as suggested by Bertrand and Anisimov, ${ }^{3}$ was incorporated in the regular part of the chemical potential in our approach.

\section{Appendix B: Isochoric heat capacity in scaling models}

Equations (11)-(13) can be generalized as

$$
\begin{aligned}
& h_{1}=c \Delta \hat{T}+a \Delta \hat{P}, \\
& h_{2}=-d \Delta \hat{P}+b \Delta \hat{T}, \\
& h_{3}=\Delta \hat{P}-\Delta \hat{\mu}+\Delta \hat{\mu}^{\mathrm{r}},
\end{aligned}
$$

The primes of the parameters $a^{\prime}$ and $b^{\prime}$ have been omitted for brevity. With $a=b=0$ and $c=d=1$ we recover the pure lattice-liquid model, whereas with $a=b=1$ and $c=d=0$ we obtain the pure lattice-gas model. The case $c=0$ for the lattice-gas model corresponds to $h_{1}=\Delta \hat{P}=\Delta \hat{\mu}$, as discussed in Ref. 3. The expression for the isochoric heat capacity $\hat{C}_{V}$,

$$
\hat{C}_{V}=\hat{C}_{P}-\hat{V} \hat{T} \frac{\hat{\alpha}_{V}^{2}}{\hat{\kappa}_{T}}
$$

leads to the critical part of $\hat{C}_{V}$,

$$
\frac{\hat{C}_{V}}{\hat{T}}=A \frac{\chi_{1} \chi_{2}-\chi_{12}^{2}}{a^{2} \chi_{1}-2 a d \chi_{12}+d^{2} \chi_{2}},
$$

with $A=(a b+c d)^{2}$. For the pure lattice liquid, we obtain

$$
\frac{\hat{C}_{V}}{\hat{T}}=\chi_{1}-\frac{\chi_{12}^{2}}{\chi_{2}}
$$

and for the pure lattice gas,

$$
\frac{\hat{C}_{V}}{\hat{T}}=\chi_{2}-\frac{\chi_{12}^{2}}{\chi_{1}},
$$

as obtained by Behnejad et al. ${ }^{81}$ In our model, the coefficient $a$ is small but finite and $\hat{C}_{V}$ is given by

$$
\frac{\hat{C}_{V}}{\hat{T}}=\frac{\chi_{1} \chi_{2}-\chi_{12}^{2}}{a^{2} \chi_{1}-2 a \chi_{12}+\chi_{2}} .
$$

\section{Appendix C: Adjustment of experimental data}

The density data of Mishima show systematic differences of up to $5 \mathrm{~kg} / \mathrm{m}^{3}$ with the densities reported by Asada et $a l .{ }^{20}$ with IAPWS-95 (in the region where it is reliable), and with the equation of state of Saul and Wagner. ${ }^{140}$ The differences appear to be mostly pressure dependent and only slightly temperature dependent; see Fig. 37. In the range from $273 \mathrm{~K}$ to $373 \mathrm{~K}$ and $0 \mathrm{MPa}$ to $380 \mathrm{MPa}$, where the three other data sets (Asada et al., IAPWS-95, Saul and Wagner) overlap and can be considered reliable, their maximum mutual difference is $0.8 \mathrm{~kg} / \mathrm{m}^{3}$. Therefore, we decided that a correction of Mishima's data is justified. A pressure-dependent density correction was determined by fitting a quadratic function of the pressure to the difference of Mishima's densities and the correlation ${ }^{141}$ of Asada et al., between $245 \mathrm{~K}$ and $274 \mathrm{~K}$ (Fig. 37). This density correction was then subtracted from all densities measured by Mishima.

Because of an incorrect graph, the heat-capacity data shown in the article of Bertolini et al. ${ }^{36}$ are about $4 \%$ too low. The discrepancy was noted by Duan et al., ${ }^{142}$ but they did not apply a correction. Tombari et al. ${ }^{35}$ and Mishima ${ }^{21}$ corrected the data without comment. A quantitative correction can be obtained because the graph of Bertolini et al. also contains a plot of an equation of Leyendekkers and Hunter, ${ }^{143}$ and the data in the graph can be scaled until the curve matches the equation. The corrected heat capacity $C_{P}^{\text {corr }}$ may be calculated from the original $C_{P}^{\mathrm{org}}$ by $C_{P}^{\mathrm{corr}}=13.74+0.863 C_{P}^{\mathrm{org}}$, with $C_{P}$ in $\mathrm{J} \mathrm{mol}^{-1} \mathrm{~K}^{-1}$. This correction is slightly smaller than that of Tombari et al. and of Mishima.

\footnotetext{
${ }^{1}$ P. G. Debenedetti, J. Phys.: Condens. Matter 15, R1669 (2003).
} 


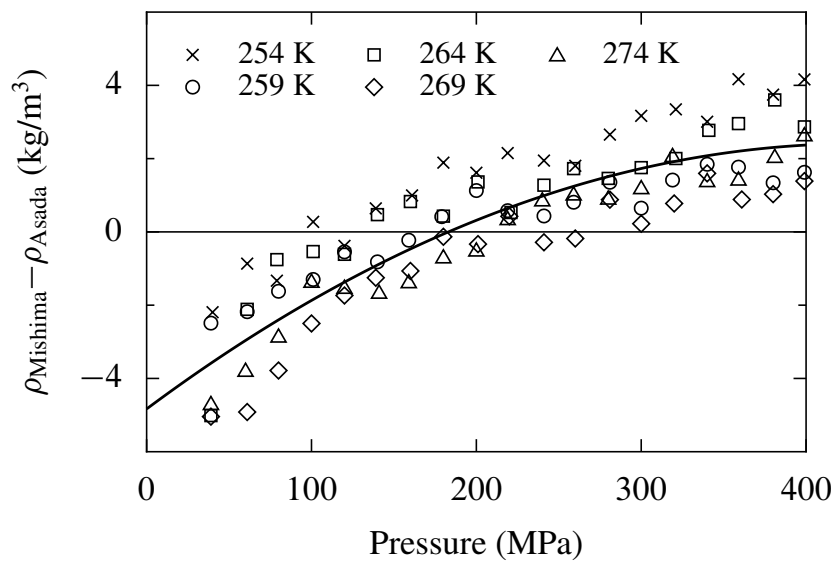

FIG. 37. Difference of Mishima's ${ }^{21}$ density and the correlation of Asada et al. ${ }^{20}$ The curve is a quadratic fit, $\Delta \rho=-4.83+0.03348 P-$ $3.87 \times 10^{-5} P^{2}$, with $\Delta \rho$ in $\mathrm{kg} / \mathrm{m}^{3}$ and pressure $P$ in $\mathrm{MPa}$.

${ }^{2}$ P. H. Poole, F. Sciortino, U. Essmann, and H. E. Stanley, Nature (London) 360, 324 (1992).

${ }^{3}$ C. E. Bertrand and M. A. Anisimov, J. Phys. Chem. B 115, 14099 (2011).

${ }^{4}$ C. A. Jeffery and P. H. Austin, J. Chem. Phys. 110, 484 (1999).

${ }^{5}$ S. B. Kiselev, Int. J. Thermophys. 22, 1421 (2001).

${ }^{6}$ S. B. Kiselev and J. F. Ely, J. Chem. Phys. 116, 5657 (2002).

${ }^{7}$ D. A. Fuentevilla and M. A. Anisimov, Phys. Rev. Lett. 97, 195702 (2006), erratum ibid. 98, 149904 (2007).

${ }^{8}$ J. Kalová and R. Mareš, Int. J. Thermophys. 31, 756 (2010).

${ }^{9}$ D. T. Limmer and D. Chandler, J. Chem. Phys. 135, 134503 (2011).

${ }^{10}$ K. T. Wikfeldt, A. Nilsson, and L. G. M. Pettersson, Phys. Chem. Chem. Phys. 13, 19918 (2011).

${ }^{11}$ Revised Release on the IAPWS Formulation 1995 for the Thermodynamic Properties of Ordinary Water Substance for General and Scientific Use, International Association for the Properties of Water and Steam (2009), http://www.iapws.org/relguide/IAPWS95-Rev.pdf.

${ }^{12}$ W. Wagner and A. Pruß, J. Phys. Chem. Ref. Data 31, 387 (2002).

${ }^{13}$ C. A. Angell, in Water and Aqueous Solutions at Subzero Temperatures, Water: A Comprehensive Treatise, Vol. 7, edited by F. Franks (Plenum, New York, 1982) Chap. 1, pp. 1-81.

${ }^{14}$ C. A. Angell, Ann. Rev. Phys. Chem. 34, 593 (1983).

${ }^{15}$ H. Sato, K. Watanabe, J. M. H. Levelt Sengers, J. S. Gallagher, P. G. Hill, J. Straub, and W. Wagner, J. Phys. Chem. Ref. Data 20, 1023 (1991).

${ }^{16}$ R. Feistel, D. G. Wright, K. Miyagawa, A. H. Harvey, J. Hruby, D. R. Jackett, T. J. McDougall, and W. Wagner, Ocean Sci. 4, 275 (2008).

${ }^{17}$ See supplementary material at [URL will be inserted by AIP] for tables with property data for supercooled $\mathrm{H}_{2} \mathrm{O}$ and $\mathrm{D}_{2} \mathrm{O}$.

${ }^{18}$ D. E. Hare and C. M. Sorensen, J. Chem. Phys. 87, 4840 (1987).

${ }^{19}$ T. Sotani, J. Arabas, H. Kubota, and M. Kijima, High Temp. High Pressures 32, 433 (2000).

${ }^{20}$ S. Asada, T. Sotani, J. Arabas, H. Kubota, S. Matsuo, and Y. Tanaka, J. Phys.: Condens. Matter 14, 11447 (2002).

${ }^{21}$ O. Mishima, J. Chem. Phys. 133, 144503 (2010).

${ }^{22}$ B. Guignon, C. Aparicio, and P. D. Sanz, J. Chem. Eng. Data 55, 3338 (2010).

${ }^{23}$ Revised Release on the Pressure along the Melting and Sublimation Curves of Ordinary Water Substance, IAPWS (2011), http://www.iapws. org/relguide/MeltSub2011.pdf; W. Wagner, T. Riethmann, R. Feistel, and A. H. Harvey, J. Phys. Chem. Ref. Data 40, 043103 (2011).

${ }^{24}$ H. Kanno, R. J. Speedy, and C. A. Angell, Science 189, 880 (1975).

${ }^{25}$ H. Kanno and K. Miyata, Chem. Phys. Lett. 422, 507 (2006).

${ }^{26}$ L. Ter Minassian, P. Pruzan, and A. Soulard, J. Chem. Phys. 75, 3064 (1981).

${ }^{27}$ R. J. Speedy and C. A. Angell, J. Chem. Phys. 65, 851 (1976).

${ }^{28}$ H. Kanno and C. A. Angell, J. Chem. Phys. 70, 4008 (1979).

${ }^{29}$ D. E. Hare and C. M. Sorensen, J. Chem. Phys. 84, 5085 (1986).

${ }^{30}$ M. A. Anisimov, A. V. Voronel', N. S. Zaugol'nikova, and G. I. Ovodov,
JETP Lett. 15, 317 (1972)

${ }^{31}$ C. A. Angell, J. Shuppert, and J. C. Tucker, J. Phys. Chem. 77, 3092 (1973).

${ }^{32}$ D. H. Rasmussen, A. P. MacKenzie, C. A. Angell, and J. C. Tucker, Science 181, 342 (1973).

${ }^{33}$ C. A. Angell, M. Oguni, and W. J. Sichina, J. Phys. Chem. 86, 998 (1982). ${ }^{34}$ D. G. Archer and R. W. Carter, J. Phys. Chem. B 104, 8563 (2000).

${ }^{35}$ E. Tombari, C. Ferrari, and G. Salvetti, Chem. Phys. Lett. 300, 749 (1999).

${ }^{36}$ D. Bertolini, M. Cassettari, and G. Salvetti, Chem. Phys. Lett. 199, 553 (1985).

${ }^{37}$ A. M. Sirota, A. J. Grishkov, and A. G. Tomishko, Thermal Eng. 17, 90 (1970).

${ }^{38}$ J. J. Manyà, M. J. Antal, Jr., C. K. Kinoshita, and S. M. Masutani, Ind. Eng. Chem. Res. 50, 6470 (2011).

${ }^{39}$ M. Oguni and C. A. Angell, J. Chem. Phys. 78, 7334 (1983).

${ }^{40} \mathrm{P}$. T. Hacker, "Experimental values of the surface tension of supercooled water," Technical Note 2510 (National Advisory Committee for Aeronautics, 1951) http://naca.central.cranfield.ac.uk/reports/1951/naca-tn-2510. pdf.

${ }^{41}$ M. A. Floriano and C. A. Angell, J. Phys. Chem. 94, 4199 (1990), data of capillaries 4 to 7 are shown in Fig. 6.

${ }^{42}$ E. H. Trinh and K. Ohsaka, Int. J. Thermophys. 16, 545 (1995).

${ }^{43}$ Y. J. Lü and B. Wei, Appl. Phys. Lett. 89, 164106 (2006).

${ }^{44}$ IAPWS Release on Surface Tension of Ordinary Water Substance, IAPWS (1994), http://www.iapws.org/relguide/surf.pdf.

${ }^{45}$ N. B. Vargaftik, B. N. Volkov, and L. D. Voljak, J. Phys. Chem. Ref. Data 12, 817 (1983).

${ }^{46}$ R. Massoudi and A. D. King, Jr., J. Phys. Chem. 78, 2262 (1974).

${ }^{47}$ A. Taschin, R. Cucini, P. Bartolini, and R. Torre, Phil. Mag. 91, 1796 (2011).

${ }^{48}$ S. Magazú, G. Maisano, D. Majolino, F. Mallamace, and P. Migliardo, J. Phys. Chem. 93, 942 (1989).

${ }^{49}$ S. C. Santucci, D. Fioretto, L. Comez, A. Gessin, and C. Masciovecchio, Phys. Rev. Lett. 97, 225701 (2006).

${ }^{50}$ M. A. Anisimov, E. E. Gorodetskii, and Yu. F. Kiyachenko, Sov. Phys.JETP 35, 1014 (1972).

${ }^{51}$ O. Benchikh, D. Fournier, and A. C. Boccara, J. Physique 46, 727 (1985).

${ }^{52}$ P. Kumar and H. E. Stanley, J. Phys. Chem. B 115, 14269 (2011).

${ }^{53}$ E. Trinh and R. E. Apfel, J. Chem. Phys. 72, 6731 (1980).

${ }^{54}$ J.-C. Bacri and R. Rajaonarison, J. Physique Lett. 40, L403 (1979).

${ }^{55}$ J. P. Petitet, R. Tufeu, and B. Le Neindre, Int. J. Thermophys. 4, 35 (1983).

${ }^{56}$ O. Conde, J. Teixeira, and P. Papon, J. Chem. Phys. 76, 3747 (1982).

${ }^{57}$ E. Trinh and R. E. Apfel, J. Acoust. Soc. Am. 63, 777 (1978).

${ }^{58}$ E. Trinh and R. E. Apfel, J. Chem. Phys. 69, 4245 (1978).

${ }^{59}$ R. J. Speedy, J. Phys. Chem. 86, 982 (1982).

${ }^{60}$ R. J. Speedy, J. Phys.: Condens. Matter 16, 6811 (2004); P. G. Debenedetti, ibid. 16, 6815 (2004).

${ }^{61}$ O. Mishima and H. E. Stanley, Nature 392, 164 (1998).

${ }^{62}$ O. Mishima, Phys. Rev. Lett. 85, 334 (2000).

${ }^{63}$ A. R. Imre and S. J. Rzoska, Adv. Sci. Lett. 3, 527 (2010).

${ }^{64}$ P. W. Bridgman, J. Chem. Phys. 3, 597 (1935).

${ }^{65}$ B. V. Zheleznyi, Russ. J. Phys. Chem. 43, 1311 (1969).

${ }^{66}$ R. T. Emmet and F. J. Millero, J. Chem. Eng. Data 20, 351 (1975).

${ }^{67}$ H. Kanno and C. A. Angell, J. Chem. Phys. 73, 1940 (1980).

${ }^{68}$ C. A. Angell and H. Kanno, Science 193, 1121 (1976).

${ }^{69}$ D. H. Rasmussen and A. P. MacKenzie, J. Chem. Phys. 59, 5003 (1973).

${ }^{70}$ M. E. Fisher, Rev. Mod. Phys. 46, 597 (1974).

${ }^{71}$ L. P. Kadanoff, in Phase Transitions and Critical Phenomena, Vol. 5a, edited by C. Domb and M. S. Green (Academic Press, New York, 1976) pp. 1-34.

${ }^{72}$ M. E. Fisher, in Critical Phenomena, Lecture Notes in Physics, Vol. 186, edited by F. J. W. Hahne (Springer, Berlin, 1983) pp. 1-139.

${ }^{73}$ A. Pelissetto and E. Vicari, Phys. Rep. 368, 549 (2002).

${ }^{74}$ J. V. Sengers and J. G. Shanks, J. Stat. Phys. 137, 857 (2009).

${ }^{75}$ J. S. Rowlinson and F. L. Swinton, Liquids and Liquid Mixtures, 3rd ed. (Butterworth, London, 1982).

${ }^{76}$ M. A. Anisimov, E. E. Gorodetskii, V. D. Kulikov, and J. V. Sengers, Phys. Rev. E 51, 1199 (1995).

${ }^{77}$ T. D. Lee and C. N. Yang, Phys. Rev. 87, 410 (1952).

${ }^{78}$ M. E. Fisher, Rep. Progr. Phys. 30(II), 615 (1967).

${ }^{79}$ J. V. Sengers and J. M. H. Levelt Sengers, in Progress in Liquid Physics, 
edited by C. A. Croxton (Wiley, New York, 1978) Chap. 4, pp. 103-174.

${ }^{80}$ J. M. H. Levelt Sengers, B. Kamgar-Parsi, F. W. Balfour, and J. V. Sengers, J. Phys. Chem. Ref. Data 12, 1 (1983).

${ }^{81} \mathrm{H}$. Behnejad, J. V. Sengers, and M. A. Anisimov, in Applied Thermodynamics of Fluids, edited by A. R. H. Goodwin, J. V. Sengers, and C. J. Peters (RSC Publishing, Cambridge, UK, 2010) Chap. 10, pp. 321-367.

${ }^{82}$ M. A. Anisimov, S. B. Kiselev, J. V. Sengers, and S. Tang, Physica A 188, 487 (1992).

${ }^{83}$ M. A. Anisimov, V. A. Agayan, and P. J. Collings, Phys. Rev. E 57, 582 (1998).

${ }^{84}$ M. E. Fisher, in Proceedings of the International school of Physics "Enrico Fermi", edited by M. S. Green (Academic Press, New York, 1971) pp. 199.

${ }^{85}$ E. Brézin, D. J. Wallace, and K. G. Wilson, Phys. Rev. Lett. 29, 591 (1972).

${ }^{86}$ P. Schofield, Phys. Rev. Lett. 22, 606 (1969).

${ }^{87}$ P. Schofield, J. D. Litster, and J. T. Ho, Phys. Rev. Lett. 23, 1098 (1969).

${ }^{88}$ P. C. Hohenberg and M. Barmatz, Phys. Rev. A 6, 289 (1972).

${ }^{89}$ M. R. Moldover, J. V. Sengers, R. W. Gammon, and R. J. Hocken, Rev. Mod. Phys. 51, 79 (1979).

${ }^{90}$ P. J. Mohr, B. N. Taylor, and D. B. Newell, "The 2010 CODATA recommended values of the fundamental physical constants," http://physics.nist.gov/constants (2011).

${ }^{91}$ Guideline on the Use of Fundamental Physical Constants and Basic Constants of Water, IAPWS (2008), http://www.iapws.org/relguide/fundam. pdf.

${ }^{92}$ E. W. Lemmon and R. Span, in Applied Thermodynamics of Fluids, edited by A. R. H. Goodwin, J. V. Sengers, and C. J. Peters (RSC Publishing, Cambridge, UK, 2010) Chap. 12, pp. 394-432.

${ }^{93}$ S. J. Henderson and R. J. Speedy, J. Phys. Chem. 91, 3069 (1987).

${ }^{94}$ D. R. Caldwell, Deep-Sea Res. 25, 175 (1978).

${ }^{95}$ S. J. Henderson and R. J. Speedy, J. Phys. Chem. 91, 3062 (1987).

${ }^{96}$ R. C. Dougherty, Chem. Phys. 298, 307 (2004).

${ }^{97}$ D. M. Murphy and T. Koop, Q. J. R. Meteorol. Soc. 131, 1539 (2005).

${ }^{98}$ M. Zábranský, Z. Kolská, V. Růžiččka, Jr., and E. S. Domalski, J. Phys. Chem. Ref. Data 39, 013103 (2010).

${ }^{99}$ G. S. Kell, J. Chem. Eng. Data 12, 66 (1967).

${ }^{100}$ C.-T. Chen and F. J. Millero, J. Acoust. Soc. Am. 62, 553 (1977).

${ }^{101}$ W. Marczak, Acustica 83, 473 (1997).

${ }^{102}$ B. Kamgar-Parsi, J. M. H. Levelt Sengers, and J. V. Sengers, J. Phys. Chem. Ref. Data 12, 513 (1983).

${ }^{103}$ A. Kostrowicka Wyczalkowska, Kh. S. Abdulkadirova, M. A. Anisimov, and J. V. Sengers, J. Chem. Phys. 113, 4985 (2000).

${ }^{104}$ W. C. Röntgen, Ann. Phys. (Leipzig) 281, 91 (1892).

${ }^{105}$ E. G. Ponyatovskii, V. V. Sinitsyn, and T. A. Pozdnyakova, JETP Lett. 60, 360 (1994)

${ }^{106}$ C. T. Moynihan, Mat. Res. Soc. Symp. Proc. 455, 411 (1997).

${ }^{107}$ I. Prigogine and R. Defay, Chemical Thermodynamics (Longmans, Green \& Co., London, 1954).

${ }^{108} \mathrm{~J}$. Hrubý and V. Holten, "A two-structure model of thermodynamic properties and surface tension of supercooled water," in Proceedings of the 14th International Conference on the Properties of Water and Steam (Maruzen, 2004); V. Holten, From supersaturated water vapour to supercooled liquid water: analysis and experiments, Master's thesis, Eindhoven University of Technology (2004).

${ }^{109}$ O. Mishima, J. Chem. Phys. 100, 5910 (1994).

${ }^{110}$ E. Whalley, D. D. Klug, and Y. P. Handa, Nature 342, 782 (1989).
${ }^{111}$ O. Mishima and H. E. Stanley, Nature 396, 329 (1998).

${ }^{112}$ Y. Zhang, A. Faraone, W. A. Kamitakahara, K.-H. Liu, C.-Y. Mou, J. B. Leão, S. Chang, and S.-H. Chen, Proc. Natl. Acad. Sci. U.S.A. 108, 12206 (2011).

$113 \mathrm{~J}$. W. Tester and M. Modell, Thermodynamics and Its Applications, 3rd ed. (Prentice Hall, Upper Saddle River, 1997).

${ }^{114}$ J. P. O'Connell and J. M. Haile, Thermodynamics: Fundamentals for Applications (Cambridge University Press, Cambridge, MA, 2005).

${ }^{115}$ M. E. Fisher, S.-Y. Zinn, and P. J. Upton, Phys. Rev. B 59, 14533 (1999).

${ }^{116}$ E. B. Moore and V. Molinero, Nature 479, 506 (2011).

${ }^{117}$ D. Liu, Y. Zhang, C.-C. Chen, C.-Y. Mou, P. H. Poole, and S.-H. Chen, Proc. Natl. Acad. Sci. U.S.A. 104, 9570 (2007).

${ }^{118}$ F. Mallamace, C. Branca, M. Broccio, C. Corsaro, C.-Y. Mou, and S.-H. Chen, Proc. Natl. Acad. Sci. U.S.A. 104, 18387 (2007).

${ }^{119}$ Y. Zhang, K.-H. Liu, M. Lagi, D. Liu, K. C. Littrell, C.-Y. Mou, and S.-H. Chen, J. Phys. Chem. B 113, 5007 (2009).

${ }^{120}$ F. Mallamace, Proc. Natl. Acad. Sci. U.S.A. 106, 15097 (2009).

${ }^{121}$ A. Nagoe, Y. Kanke, M. Oguni, and S. Namba, J. Phys. Chem. B 114, 13940 (2010).

${ }^{122}$ P. Gallo, M. Rovere, and S.-H. Chen, J. Phys.: Condens. Matter 22, $284102(2010)$.

${ }^{123}$ L. Xu and V. Molinero, J. Phys. Chem. B 115, 14210 (2011).

${ }^{124}$ S. Sastry, P. G. Debenedetti, F. Sciortino, and H. E. Stanley, Phys. Rev. E 53, 6144 (1996).

${ }^{125}$ L. P. N. Rebelo, P. G. Debenedetti, and S. Sastry, J. Chem. Phys. 109, 626 (1998).

${ }^{126}$ C. A. Angell, Science 319, 582 (2008).

${ }^{127}$ K. Stokely, M. G. Mazza, H. E. Stanley, and G. Franzese, Proc. Natl. Acad. Sci. U.S.A. 107, 1301 (2010).

${ }^{128}$ V. B. Henriques, N. Guisoni, M. A. Barbosa, M. Thielo, and M. C. Barbosa, Mol. Phys. 103, 3001 (2005).

${ }^{129}$ I. Brovchenko, A. Geiger, and A. Oleinikova, J. Chem. Phys. 123, 044515 (2005).

${ }^{130}$ I. Brovchenko and A. Oleinikova, Interfacial and Confined Water (Elsevier, Amsterdam, 2008).

${ }^{131}$ V. Molinero and E. B. Moore, J. Phys. Chem. B 113, 4008 (2009).

${ }^{132}$ Y. Liu, A. Z. Panagiotopoulos, and P. G. Debenedetti, J. Chem. Phys. 131, 104508 (2009).

${ }^{133}$ F. Sciortino, I. Saika-Voivod, and P. H. Poole, Phys. Chem. Chem. Phys. 13, 19759 (2011).

${ }^{134}$ S. A. Brazovskii, Sov. Phys.-JETP 41, 85 (1975).

${ }^{135}$ S. A. Brazovskii, I. E. Dzyaloshinskii, and A. R. Muratov, Sov. Phys.JETP 66, 625 (1987).

${ }^{136}$ E. I. Kats, V. V. Lebedev, and A. R. Muratov, Phys. Rep. 228, 1 (1993).

${ }^{137}$ M. A. Anisimov, Critical Phenomena in Liquids and Liquid Crystals (Gordon and Breach, Philadelphia, 1991).

${ }^{138}$ A. Gohin, C. Destrade, H. Gasparoux, and J. Prost, J. Physique 44, 427 (1983).

${ }^{139}$ M. A. Anisimov, S. A. Konev, V. I. Labko, G. L. Nikolaenko, G. I. Olefirenko, and I. K. Yudin, Mol. Cryst. Liq. Cryst. 146, 421 (1987); M. A. Anisimov, V. I. Labko, G. L. Nikolaenko, and I. K. Yudin, JETP Lett. 45, 111 (1987).

${ }^{140}$ A. Saul and W. Wagner, J. Phys. Chem. Ref. Data 18, 1537 (1989).

${ }^{141}$ There is a sign error in the correlation of Asada et al.: ${ }^{20}$ the coefficient $a_{6}$ in their Table 1 should have a positive sign

${ }^{142}$ F. Duan, I. Thompson, and C. A. Ward, J. Phys. Chem. B 112, 8605 (2008).

${ }^{143}$ J. V. Leyendekkers and R. J. Hunter, J. Chem. Phys. 82, 1440 (1985). 